\title{
Probing Sea Quark TMD with Drell-Yan at SpinQuest/E0139 at Fermilab
}

\author{
Ming Liu \\ Los Alamos National Laboratory \\ For the SpinQuest/E1039 Collaboration
}

QCD Evolution 2019, May 13-17

Argonne National Laboratory

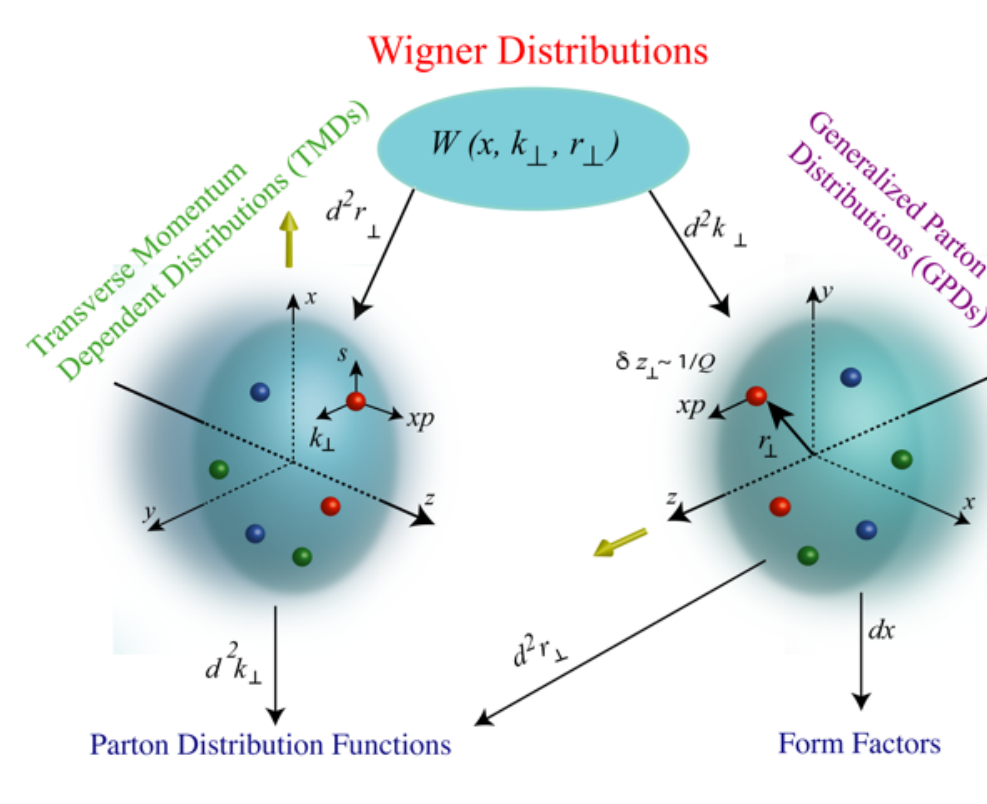




\section{Outline}

- SpinQuest/E1039 experiment at Fermilab

- E1039 polarized $\mathrm{NH}_{3} / \mathrm{ND}_{3}$ targets

- Follow up of SeaQuest/E906 unpolarized target program

- Novel physics of sea quarks at $x=0.1-0.4$

- Flavor asymmetry

- Sivers \& OAM

- Other opportunities

- Parasitic E1067 dark photon search, 2016 - 2021+

- Future E1027 polarized beam possibility, 2021+

- TMD physics complementary to the future EIC program

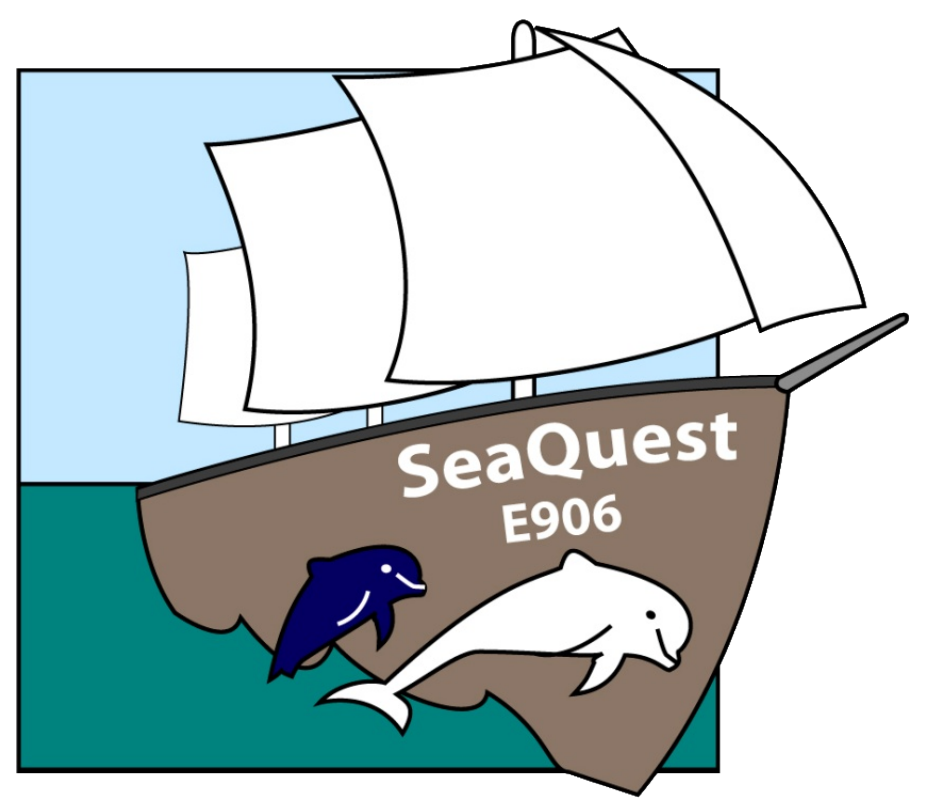

A serials of follow up experiments: SeaQuest, SpinQuest, DarkQuest

Wigner Distributions

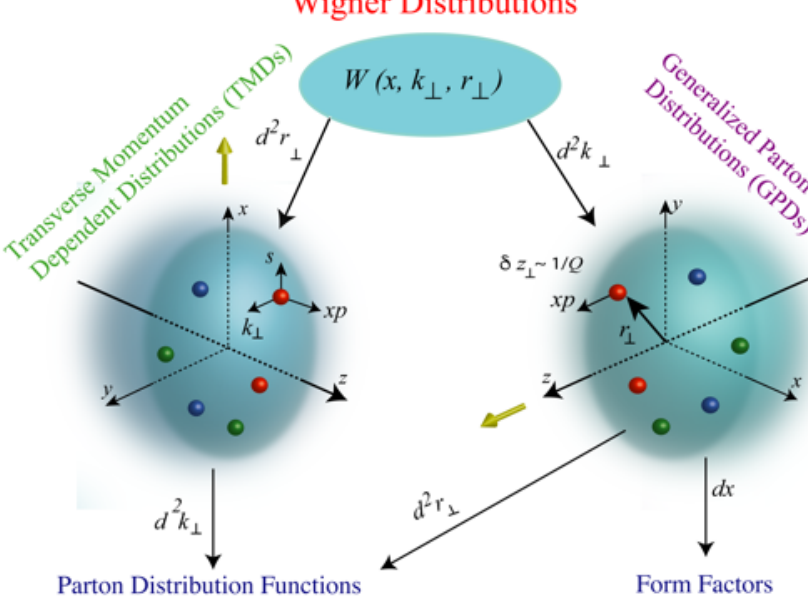


Sermitalo bis d hatensity Frontier

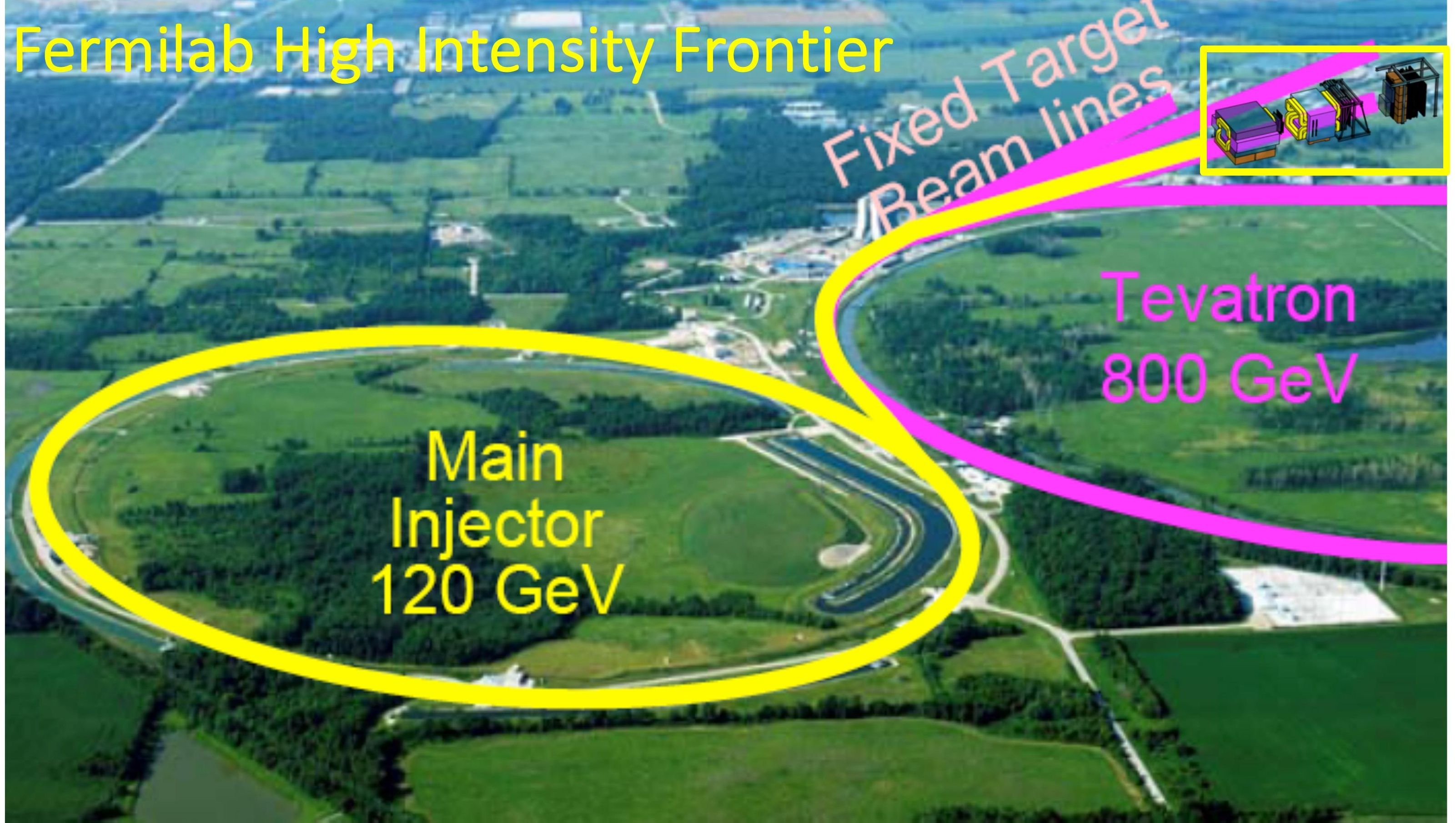




\section{SpinQuest Dimuon Spectrometer}

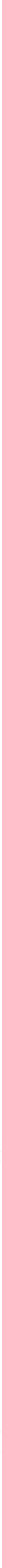




\section{Drell-Yan @SeaQuest - a Sea Quark Laboratory}

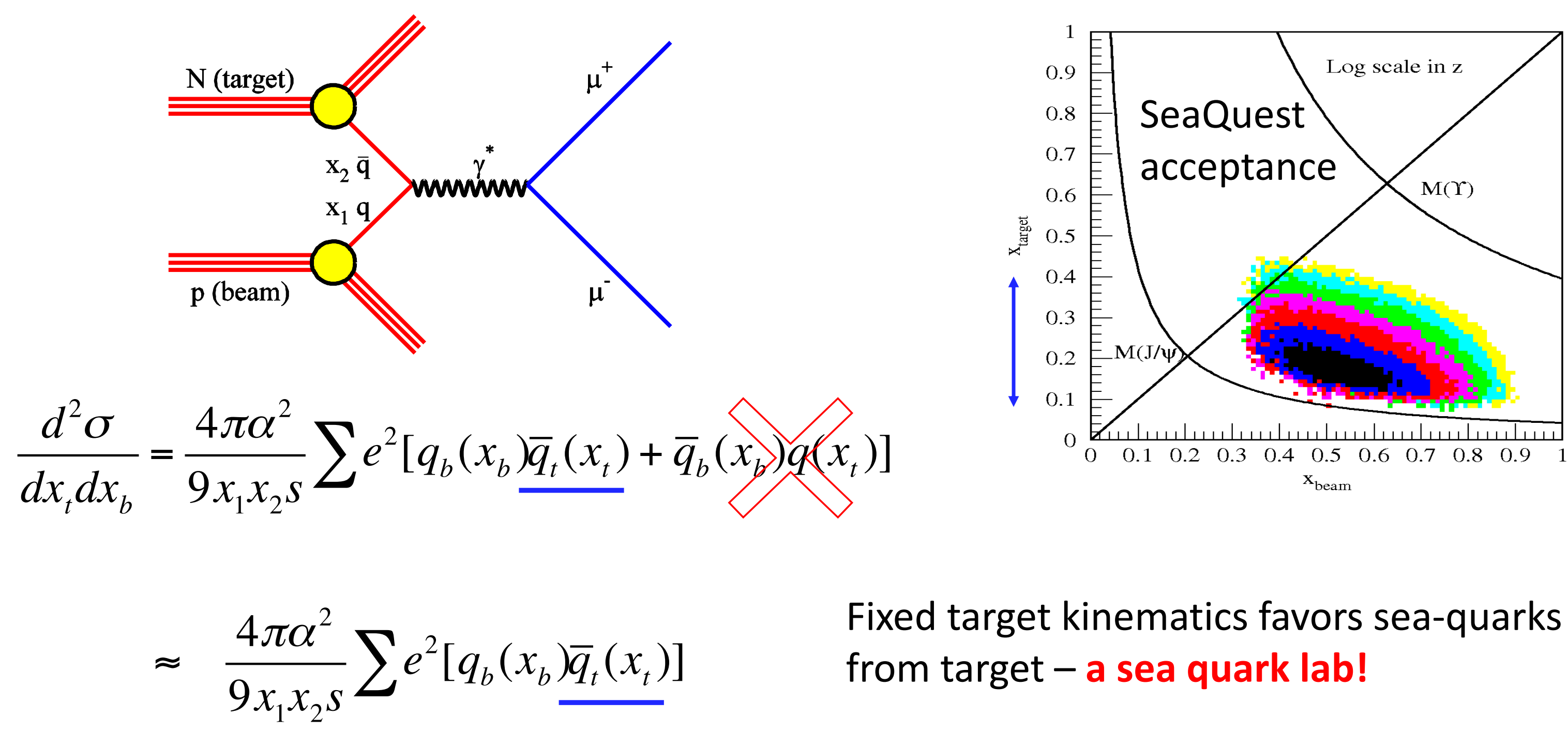




\section{Dimuon Mass from SeaQuest/E906}

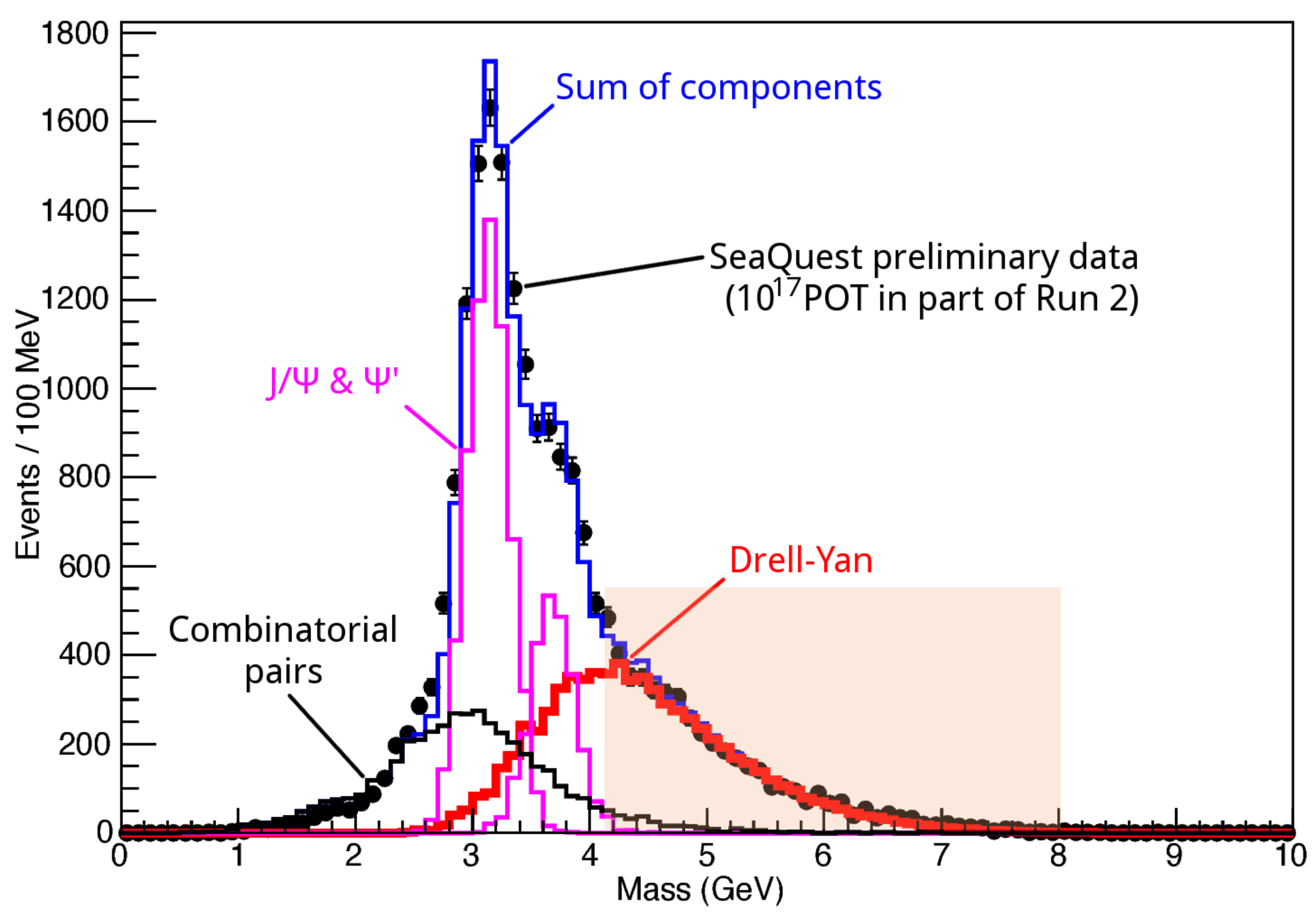




\section{E906 Unpolarized Physics Program}

- Thin targets: 10\% interaction length

- Liquid H/D

- Solid C, Fe, W

- Physics

- Sea quark flavor asymmetry, dbar/ubar

- Quark energy loss in $\mathrm{p}+\mathrm{A}$ collisions, $\mathrm{dE} / \mathrm{dx}$

- TMD and more ...

- Experimental runs -6 years

- 2012 - commissioning

- 2017 - completed

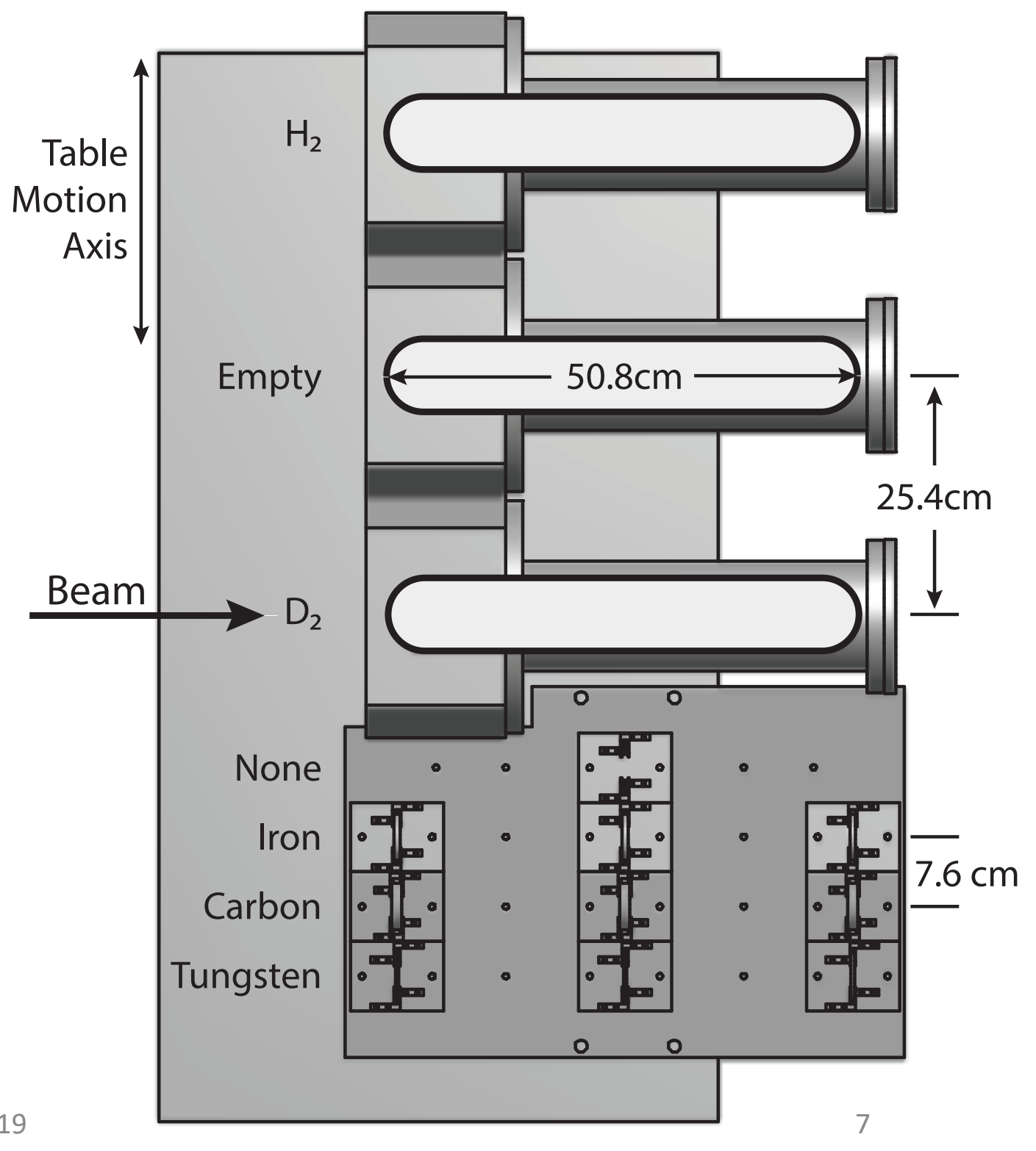




\section{Flavor Asymmetry of Sea Quarks at Intermediate x}

$$
\begin{aligned}
& \text { Proton vs "Neutron" targets: } \\
& \left.\frac{\sigma^{p d \rightarrow \mu^{+} \mu^{-}}}{\sigma^{p p \rightarrow \mu^{+} \mu^{-}}}\right|_{x_{\mathrm{b} \gg x_{\mathrm{t}}}} \approx \frac{1}{2}\left[1+\frac{\bar{d}\left(x_{\mathrm{t}}\right)}{\bar{u}\left(x_{\mathrm{t}}\right)}\right]
\end{aligned}
$$

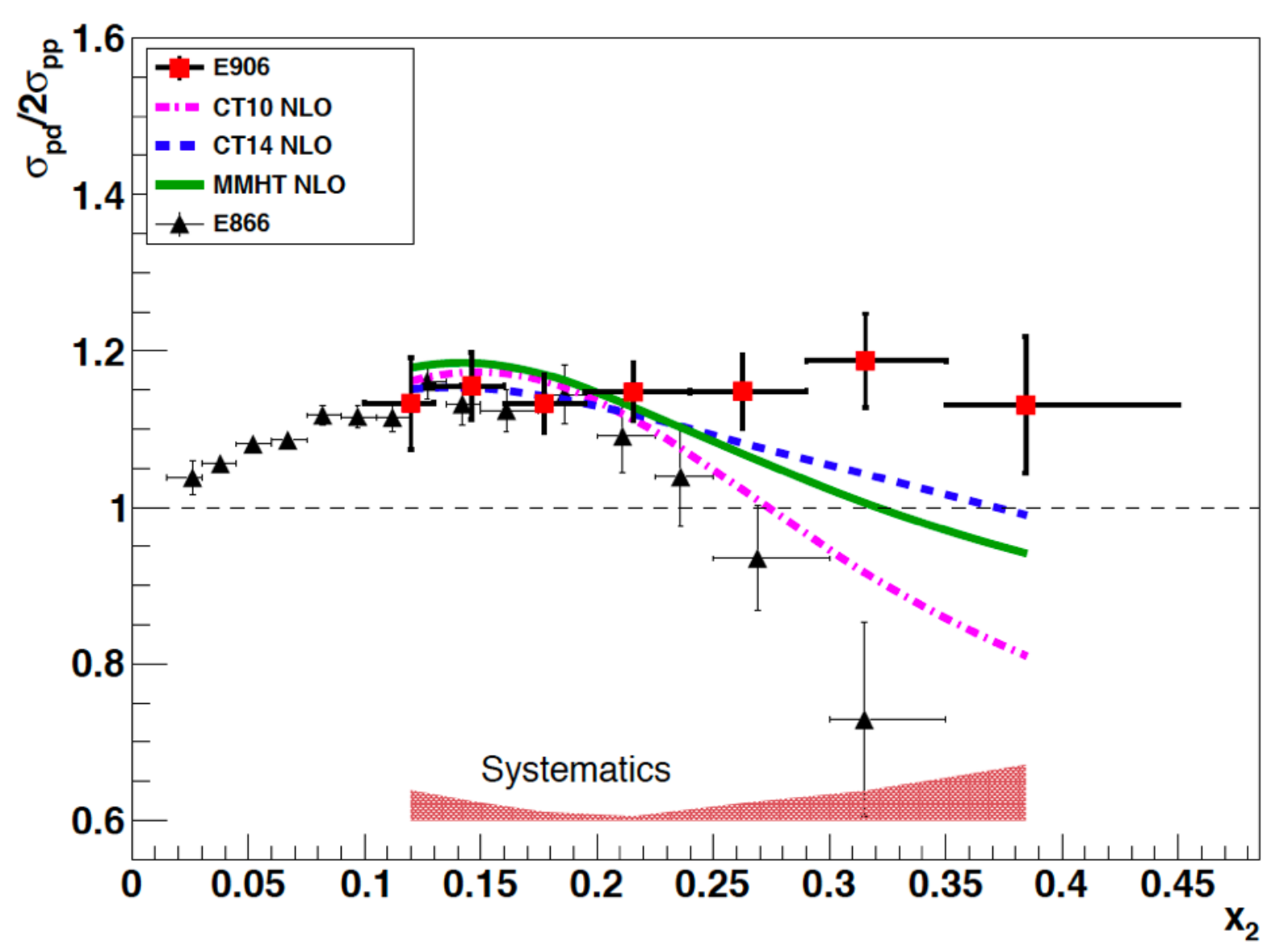




\section{Sea Quark Flavor Asymmetry and OAM}

Pion cloud model

- Sea-quark flavor asymmetry

- Sea-quark orbital angular motion

- Expect large Sivers function at $x=0.1-0.4$
Pion cloud and

Drell-Yan process

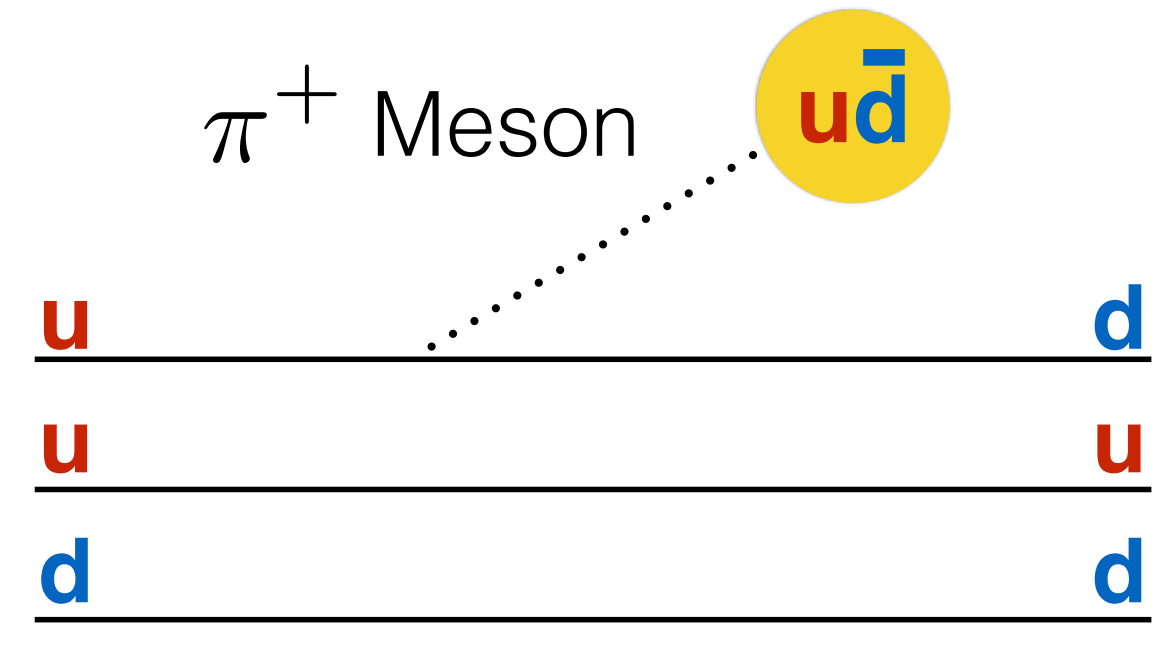

proton neutron
(4) d

virtual pion

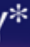

MWWWW Drell-Yan process

(u) (u 


\section{Nucleon 3-D Structure and Sivers Function}

Sivers function $f_{1 T}^{\perp}\left(\mathrm{x}, \mathrm{k}_{\mathrm{T}}\right)$

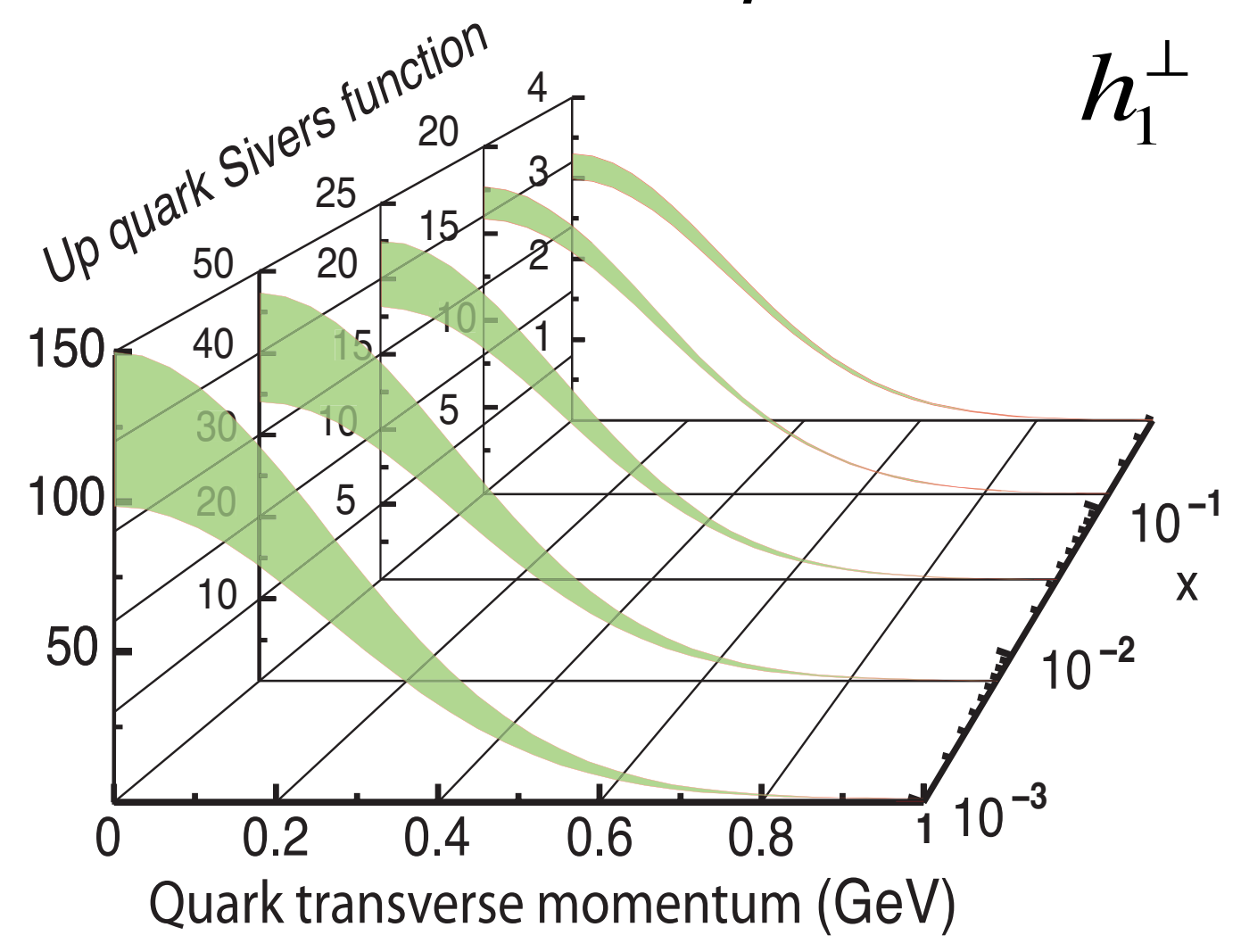

$$
f_{1 T}^{\perp}=\bigodot-\bigodot
$$

u quark

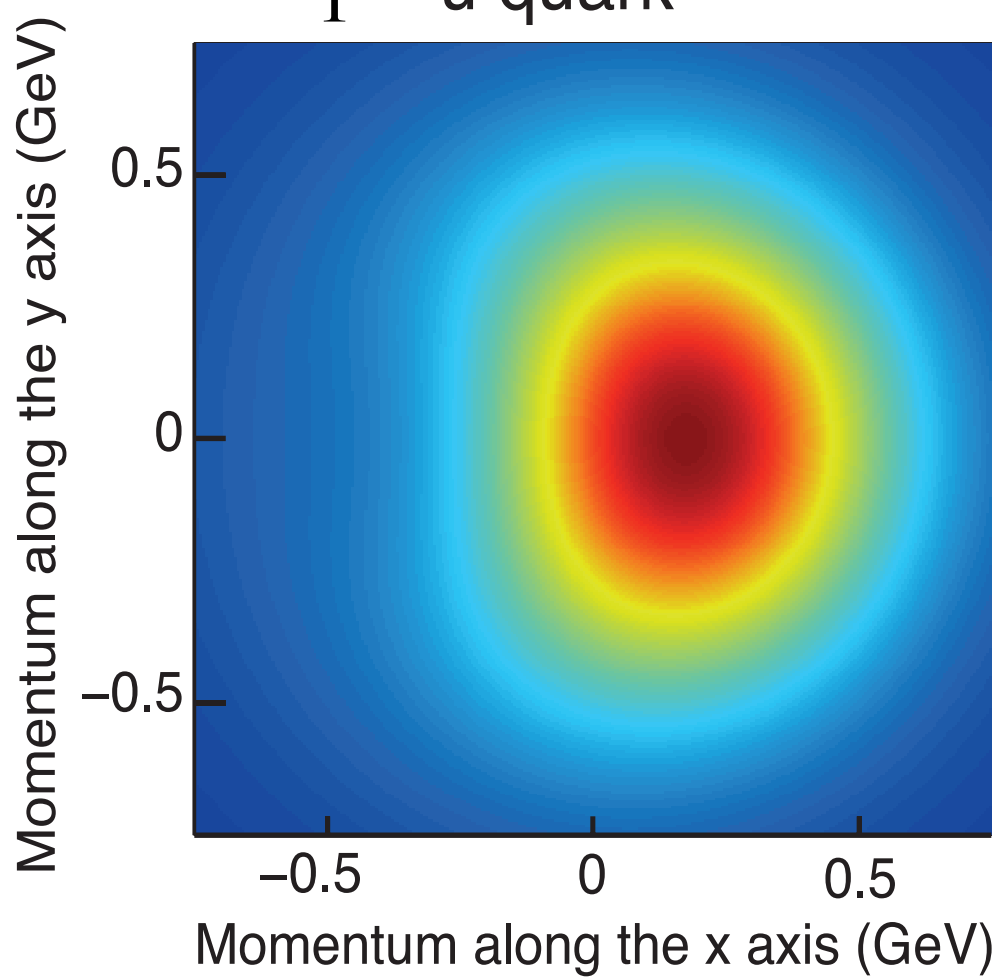




\section{Sivers Functions from Global Fits}

- Sea Quark Sivers poorly constrained, SIDIS not sensitive to sea quarks at large x

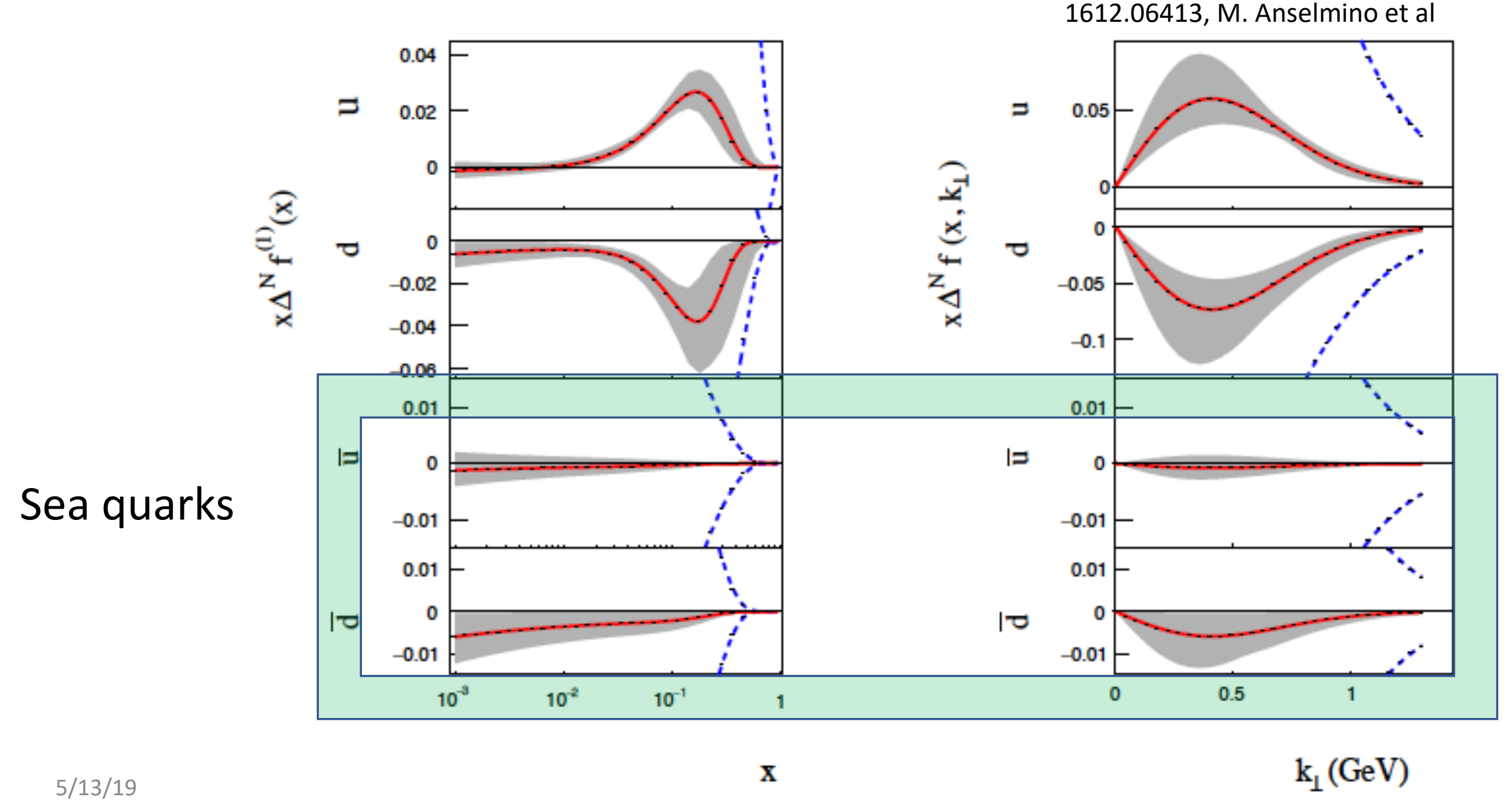




\section{RHIC pp500GeV: $W^{+/-} A_{N}$}

$$
\mathrm{A}_{N}\left(\mathrm{~W}^{+}\right) \sim\left(\Delta^{N} f_{u / p^{\dagger}} \otimes f_{\bar{d} / p}+\Delta^{N} f_{\bar{d} / p^{\uparrow}} \otimes f_{u / p}\right)
$$

$$
\mathrm{A}_{\mathrm{N}}\left(\mathrm{W}^{-}\right) \sim\left(\Delta^{N} f_{\bar{u} / p^{\uparrow}} \otimes f_{d / p}+\Delta^{N} f_{d / p^{\uparrow}} \otimes f_{\bar{u} / p}\right)
$$

\section{RHIC data:}

- A mix of valence and sea quark Sivers

- Quark flavor identified

- High Q2

- Statistically limited, O(10\%)

- Hints of sizable sea-quark Sivers contributions

\section{E1039:}

- low $Q^{2}$

- high statistics, O(1\%)

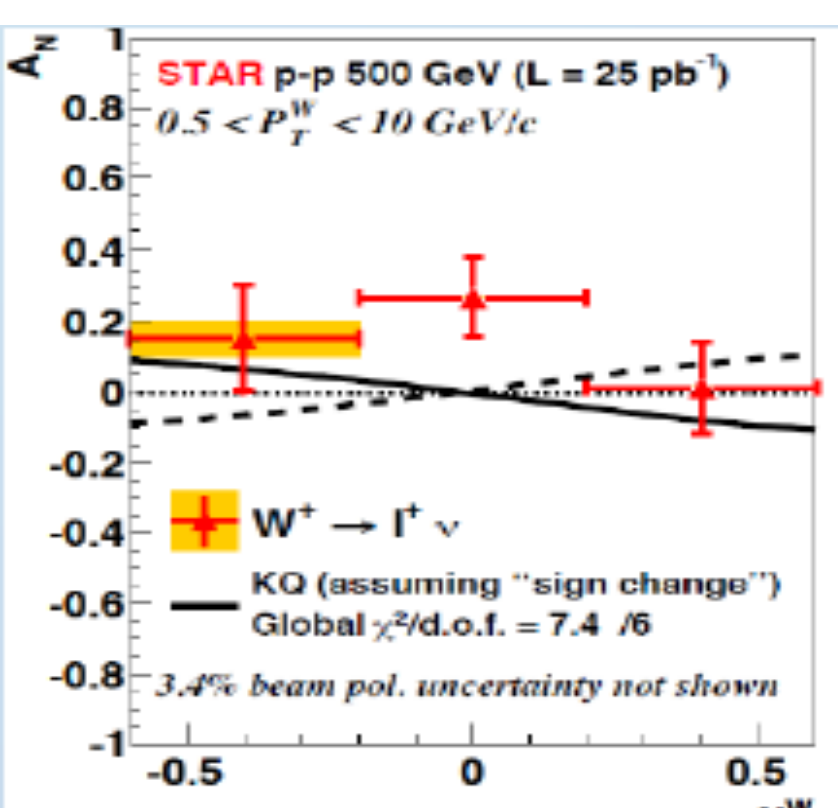

F. Tian et al. / Nuclear Physics A 968 (2017) 379-390

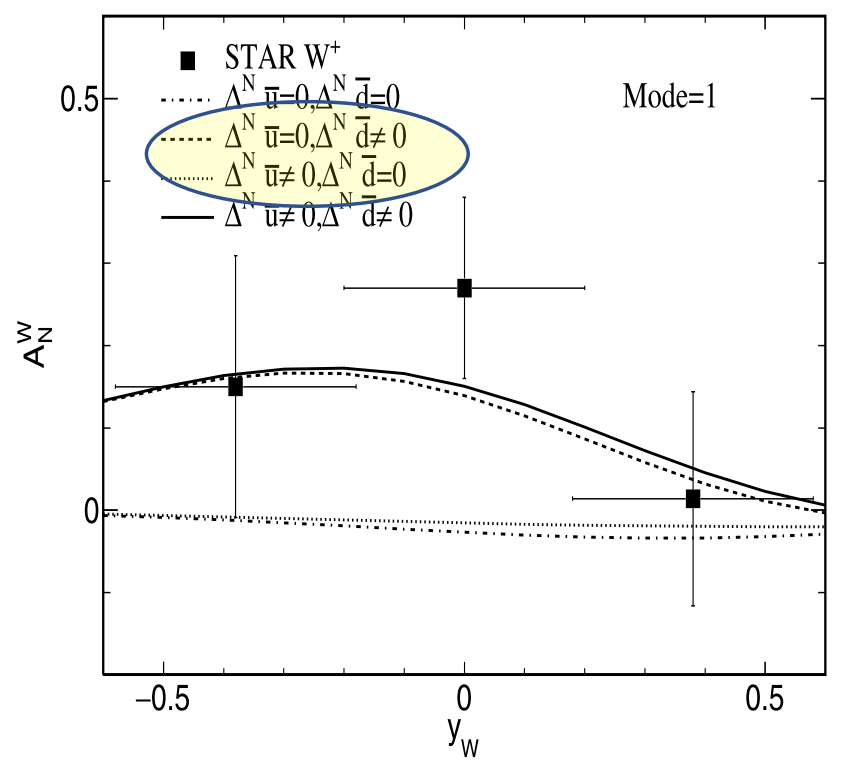

(b) $A_{W^{+}}^{N}$.
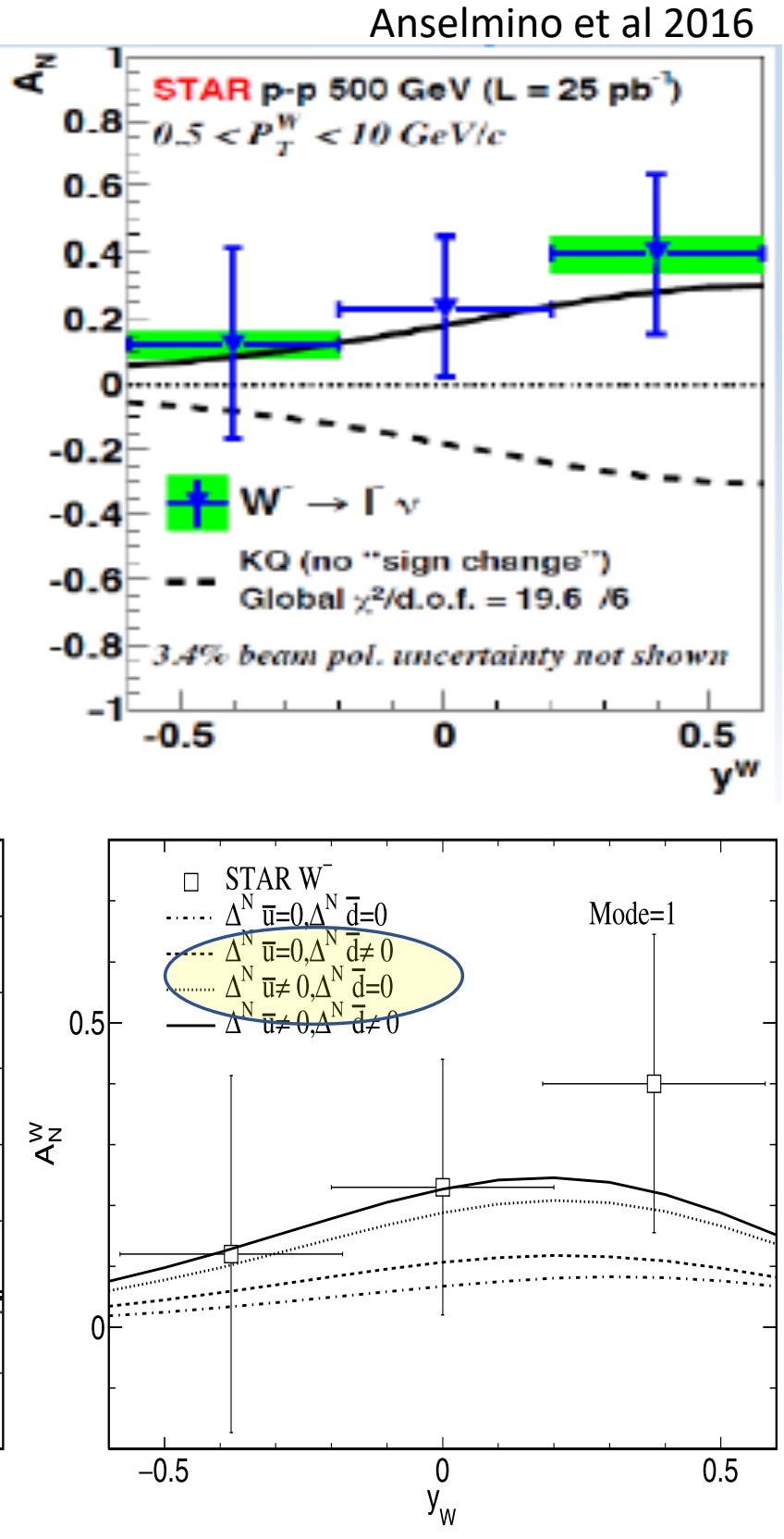

(a) $A_{W^{-}}^{N}$. 


\section{Polarized $\mathrm{NH}_{3}$ Target Developed for DY Sivers}

Microwave: Induces electron spin flips

- EIO + Power equip:

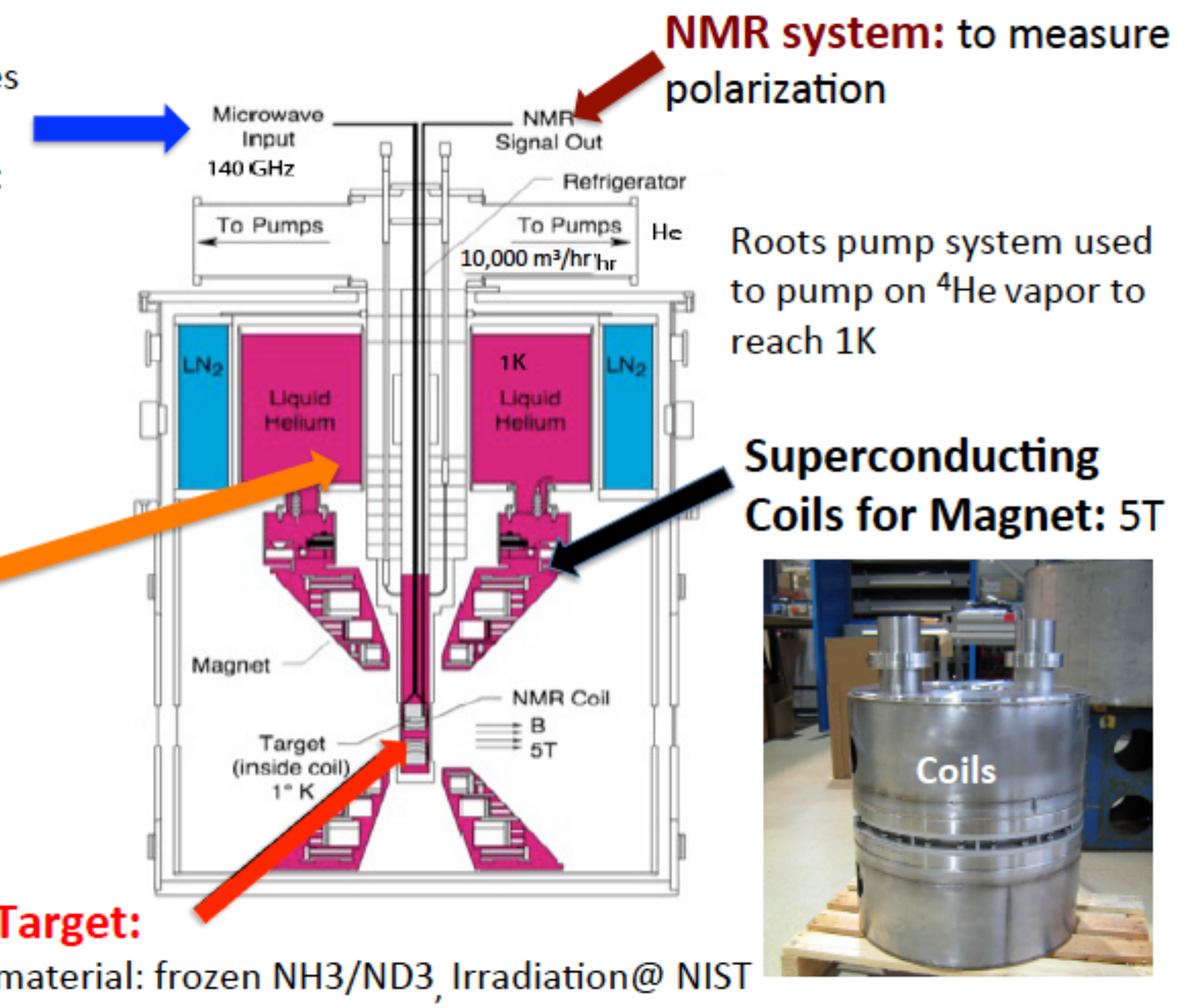




\section{Dynamic Nuclear Polarization: Pol. $90 \%$}

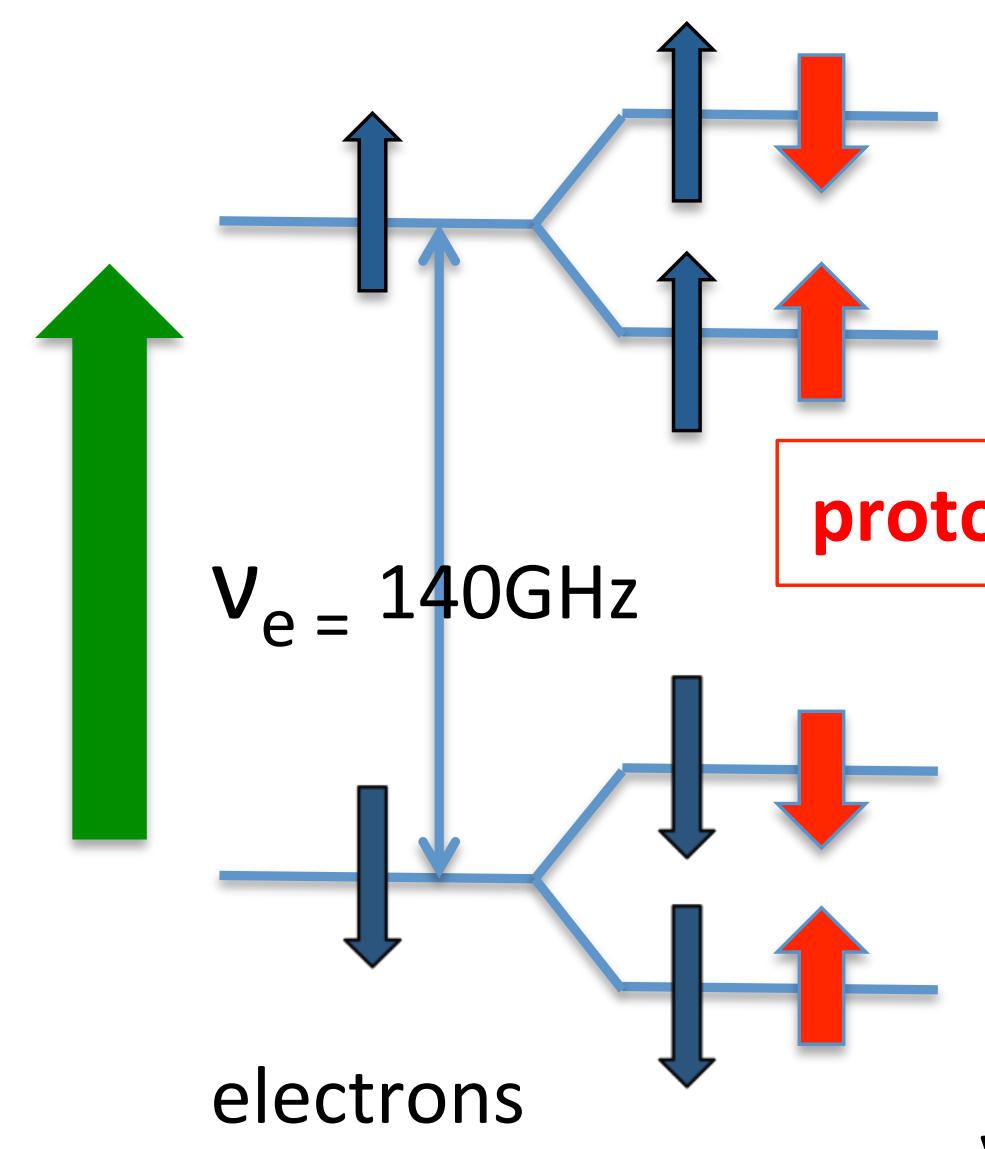

Hydrogen
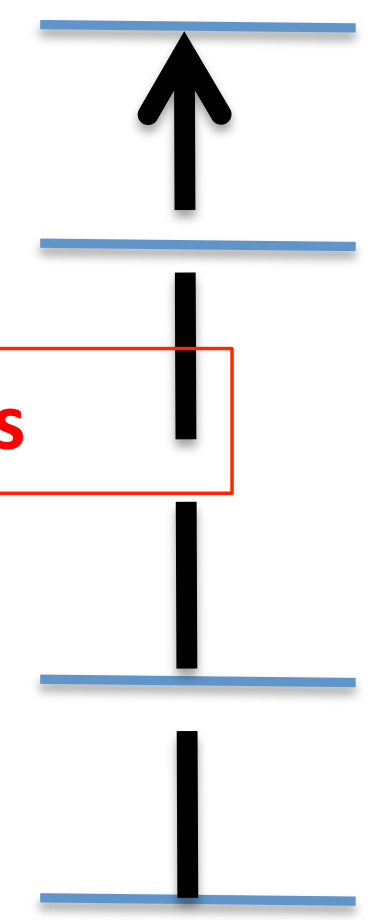

$\mathrm{v}_{\mathrm{e}}+\mathrm{v}_{\mathrm{p}}$ micro wave

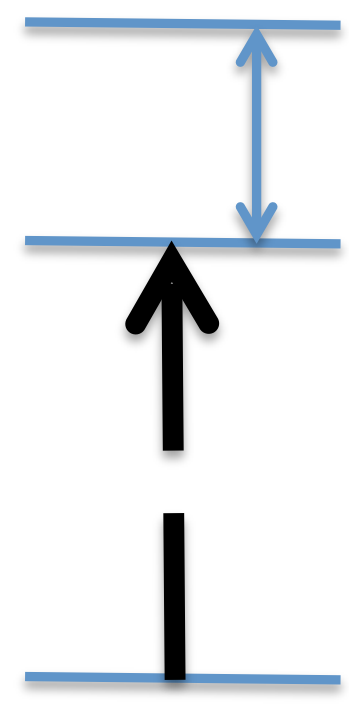

With DNP,

$$
\text { Pol. 90\% }
$$

W/o DNP, at thermal equilibrium:

$-\mathrm{T}=1 \mathrm{~K}$$$
\text { - } \mathrm{B}=5 \mathrm{~T}
$$

Proton target polarization:

$$
P_{i}=0.5 \%
$$

$\mathrm{V}_{\mathrm{e}}-\mathrm{V}_{\mathrm{p}}$ 


\section{Projected SpinQuest Target and Beam Performance}

$$
A_{\text {meas }}=f \cdot P_{\mathrm{T}} \cdot A_{\text {phy }}
$$

\begin{tabular}{|l|r||l|r|}
\hline \multicolumn{2}{|c|}{ Target } & \multicolumn{2}{c|}{ Beam } \\
\hline Polarization $P$ & $88 \%$ & Beam & $10^{13}$ p per spill \\
\hline Packing fraction & .6 & spill & $5 \mathrm{sec}$, one per minute \\
\hline Dilution Factor $f$ & .176 & Luminosity & $4^{*} 10^{35} / \mathrm{cm}^{2} / \mathrm{s}$ \\
\hline Density $\mathrm{NH}_{3}$ & $.82 \mathrm{~g} / \mathrm{cm}^{3}$ & E $_{\text {Beam }}$ & $120 \mathrm{GeV}$ \\
\hline & & Total $\mu^{+} \mu^{-}$pairs & $4.59 * 10^{5}$ \\
\hline & & Experiment available & .48 \\
\hline
\end{tabular}




\section{Projected Drell-Yan Transverse Single Spin Asymmetry}

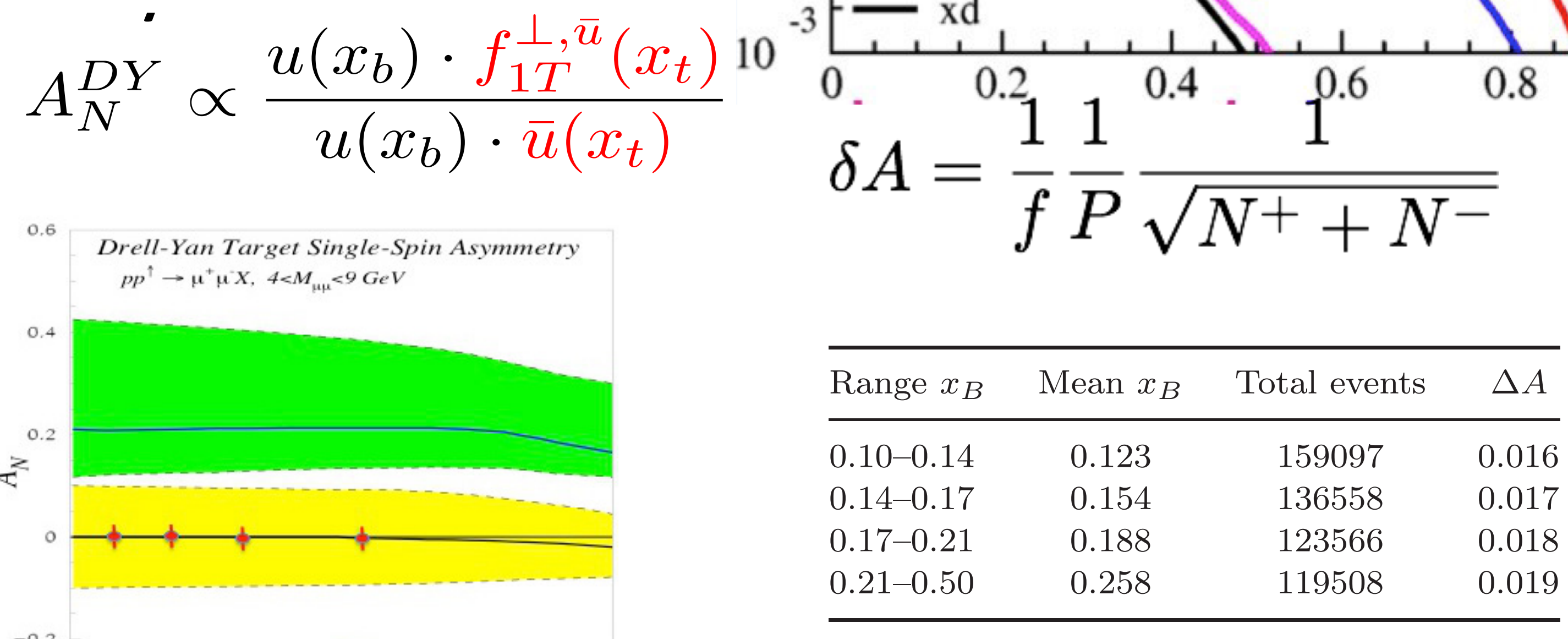




\section{Drell-Yan Sivers Asymmetries w/ QCD Evolution}

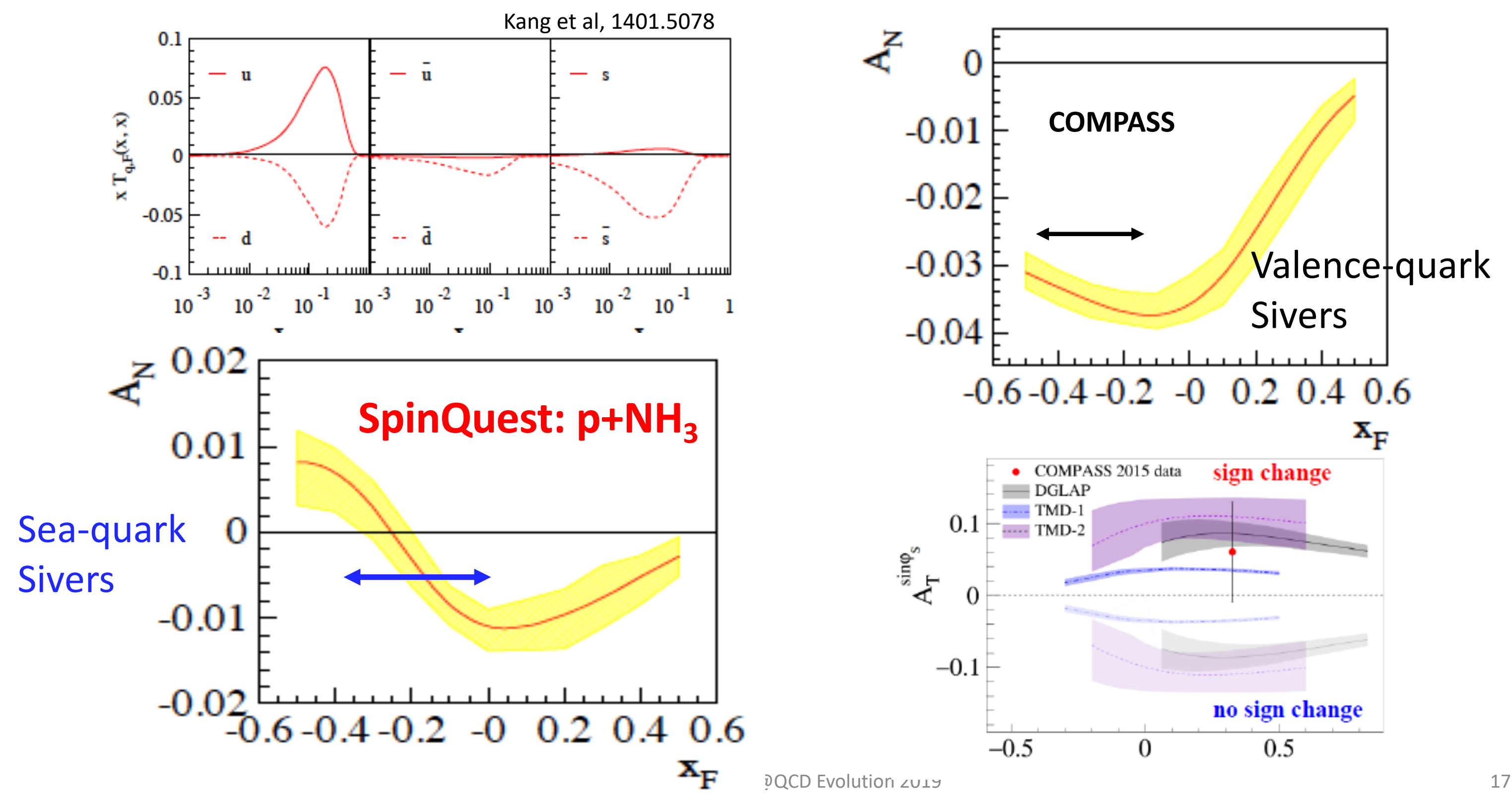




\section{E1039 Status \& Plan}

- DOE approval, March 2018

- E906 decommissioned 6/2018

- Polarized target to be installed by fall of 2019

- $\quad$ E1039 commissioning starts in late 2019

- $\quad$ Run for 2+ years, 2019-2021+

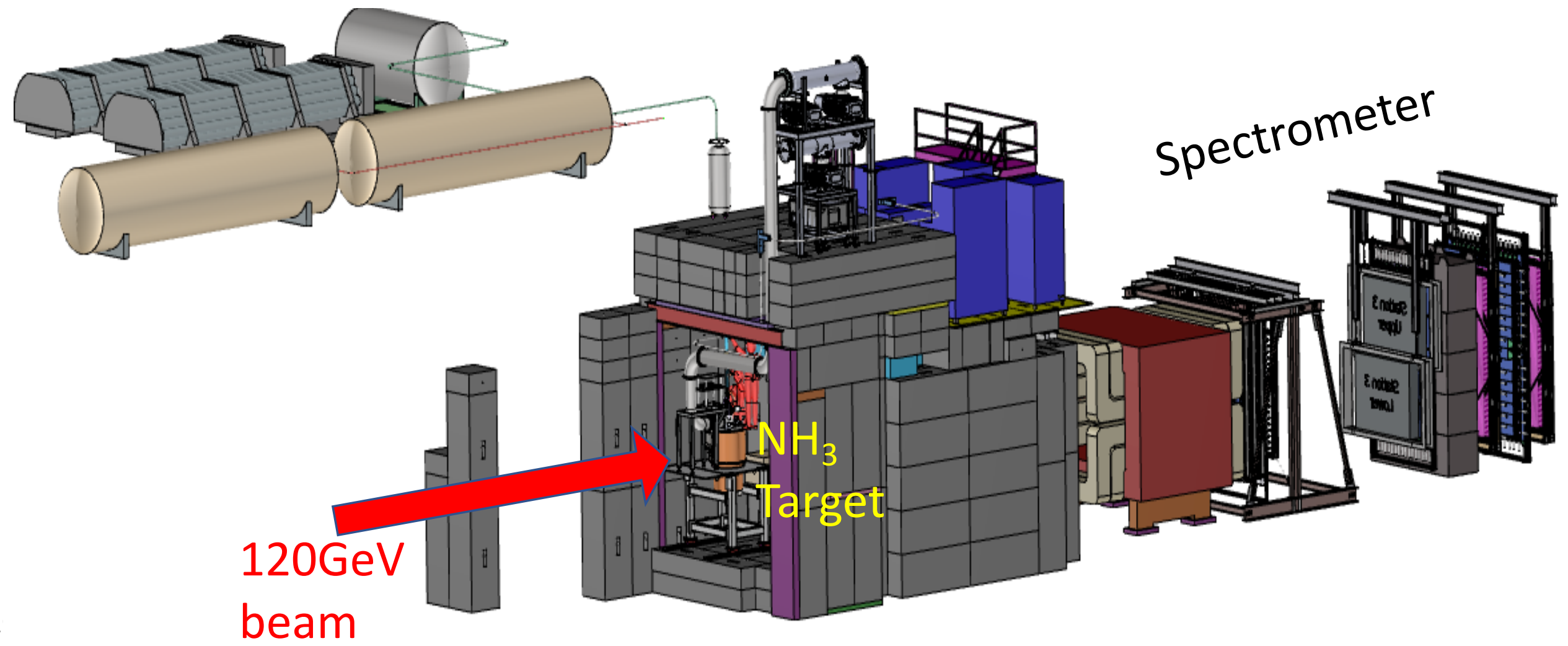




\section{Fermilab Long Range Plan}

LONG-RANGE PLAN

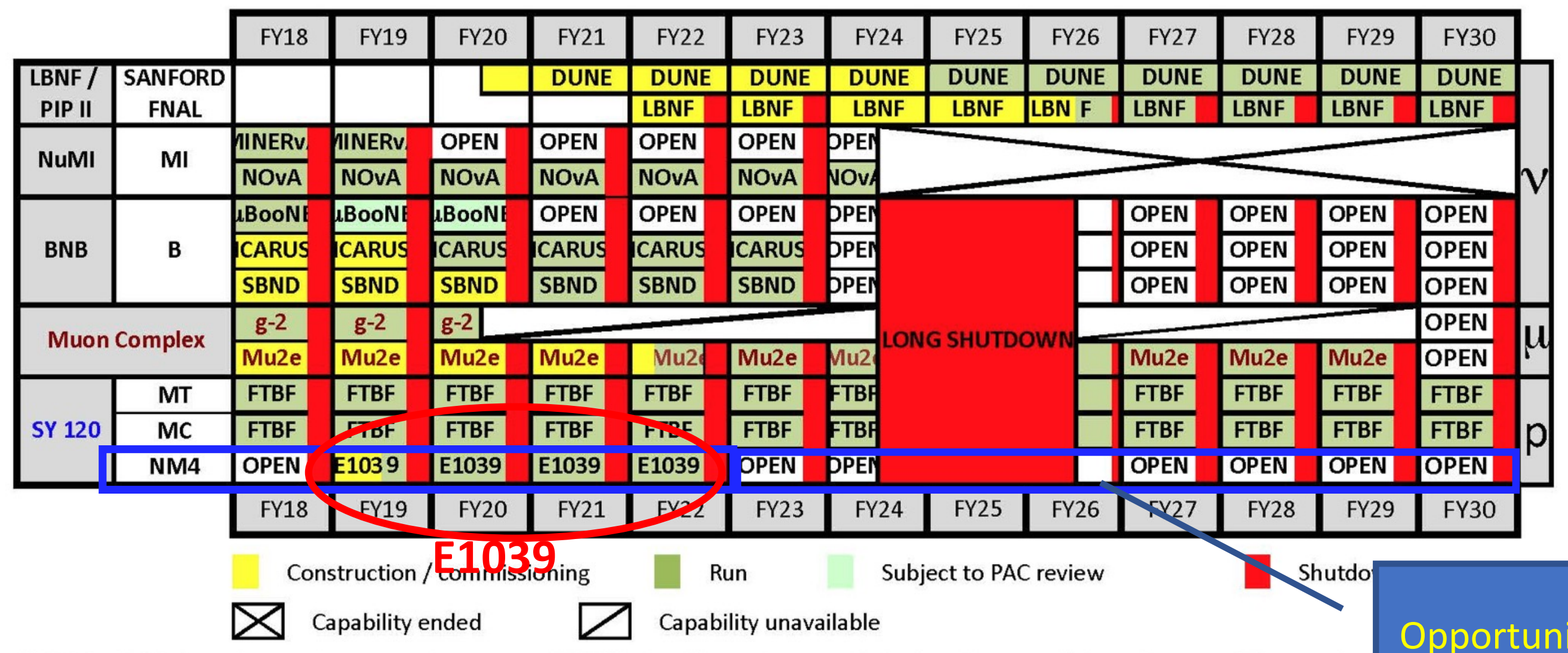

NOTES: 1 . Mu2e estimates 4 year running starts mid-FY22 after 18 months commissioning. Assume, with contingency, 5.5 years data 2. DUNE: 1st 10kT detector module commissioned in FY24. Runs without beam FY25 to mid-FY26.

3. NOvA runs as long as possible [in the spirit of PAC Nov 2017].

4. Assume NuMI in nubar mode through FY19 - facilitates 12E20 POT for MINERvA [PAC Nov 2017]. Assumption may need $r$

5. Assume g-2 completed before Mu2e commissioning start mid-FY20. Very tight. Needs scrutiny.

6. Assume E1039 fully approved \& commissioned by mid-FY19.

Experiment estimates 2 yrs run. Add 1 yr contingency. [Stage 1 approval PAC June 2013, update July 2017]

7. FY19 and FY20 MicroBooNE running subject to future PAC review [PAC July 2017]. 


\section{Physics Beyond E1039 Polarized DY $A_{N}$}

- Dark sector physics search - DarkQuest

- Parasitic run with E1039: 2019 - 2021+

- A new proposal for a dedicated run after E1039

- Physics with polarized beams - E1027

- Polarize the Main Injector $120 \mathrm{GeV}$ beam

- Valence quark Sivers

- Test QCD dynamics in DY vs DIS

- TMD physics
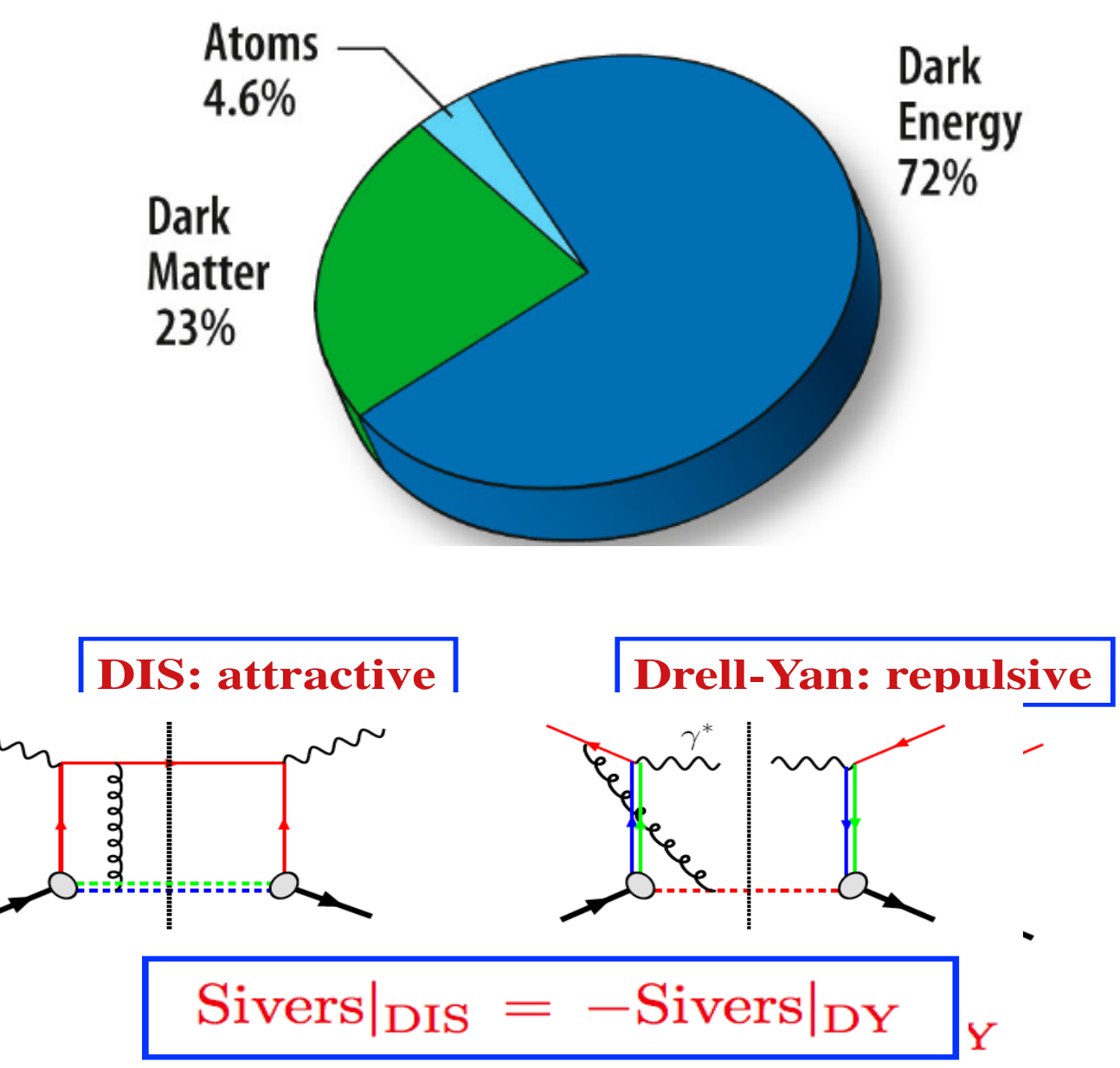
Dark Sector Physics Search at DarkQuest

2019 2021: parasitic run for DM search

2021+: a proposal for a long term DM program after E1039; parasitic TMD physics?

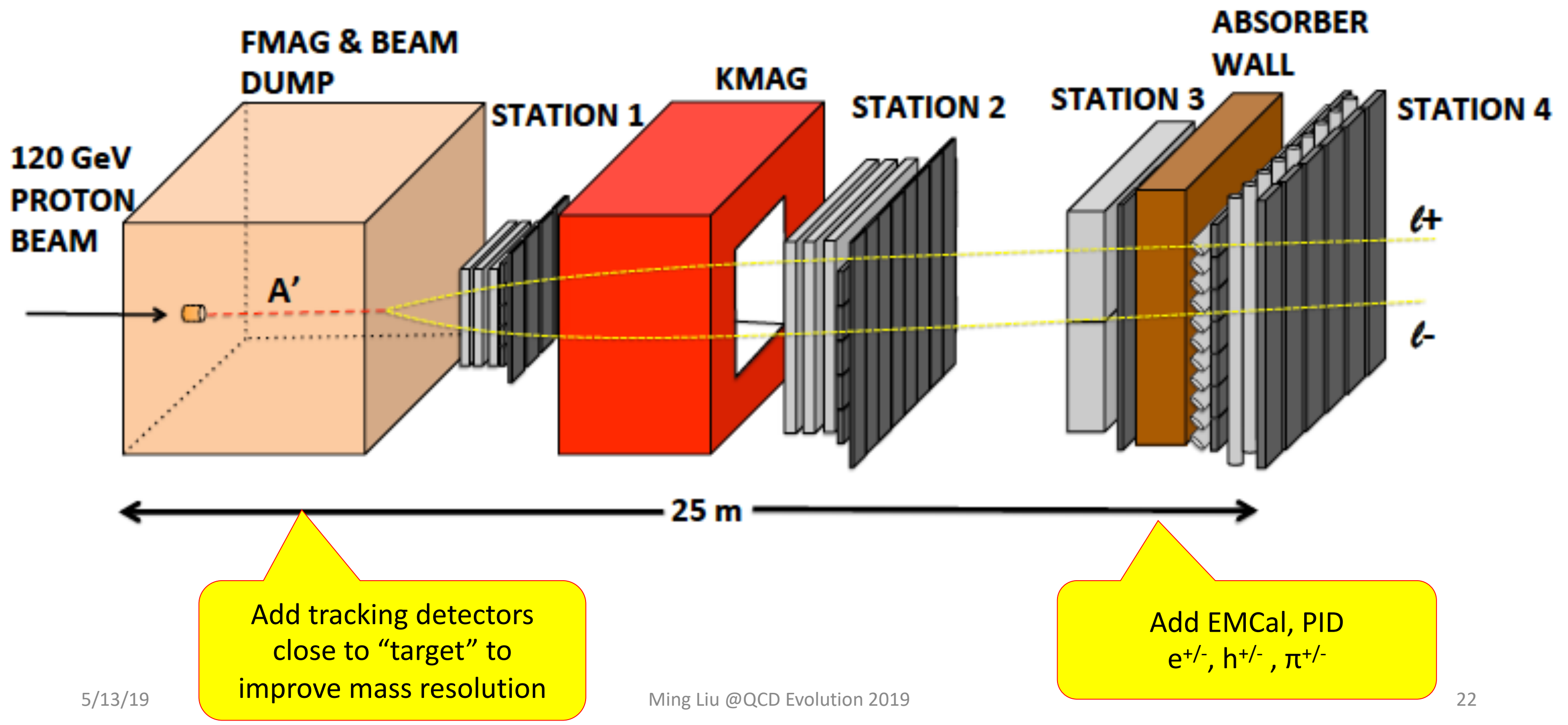




\section{Dark Photon Search at DarkQuest with Future Projections}
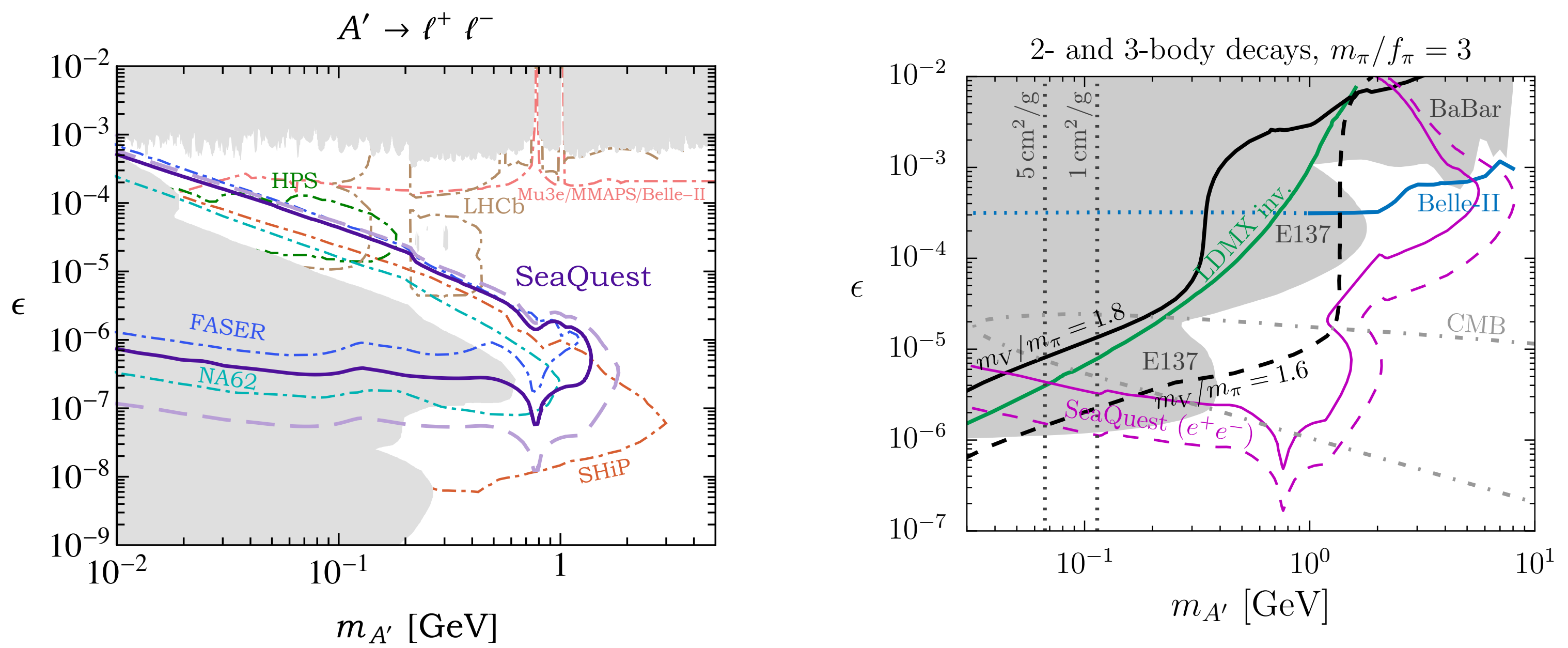


\section{Spin physics Program with Polarized Main Injector - E1027}

- Access both polarized valence and sea quarks

- Fermilab PAC stage-1 approved

- Complementary to the future EIC TMD Physics

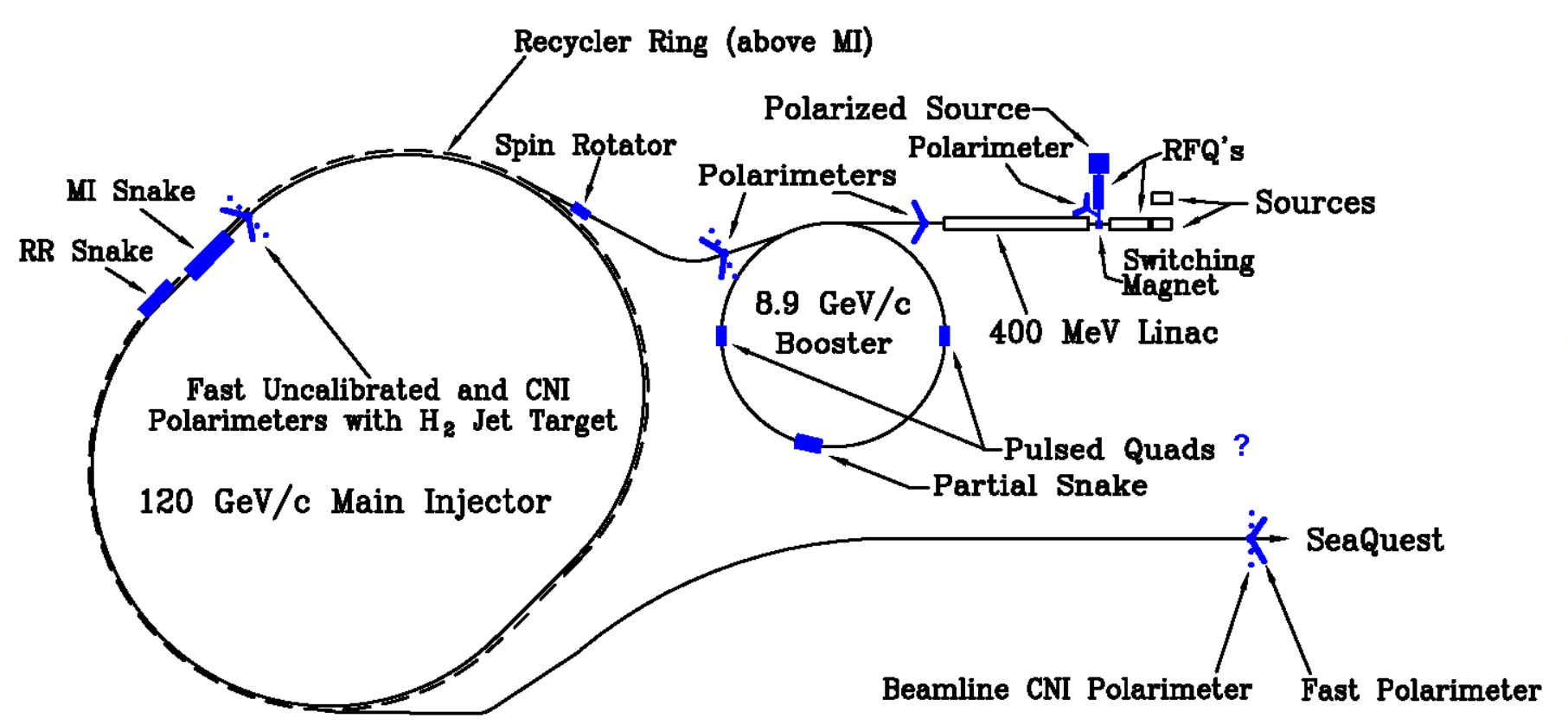

Test QCD processes in DY vs DIS over a broad range of kinematics

$$
A_{N} \equiv \frac{N^{\uparrow}-N^{\downarrow}}{N^{\uparrow}+N^{\downarrow}} \propto \frac{f_{1 T}^{\perp, u}\left(x_{B}\right) \cdot \bar{u}\left(x_{T}\right)}{u\left(x_{B}\right) \cdot \bar{u}\left(x_{T}\right)}
$$

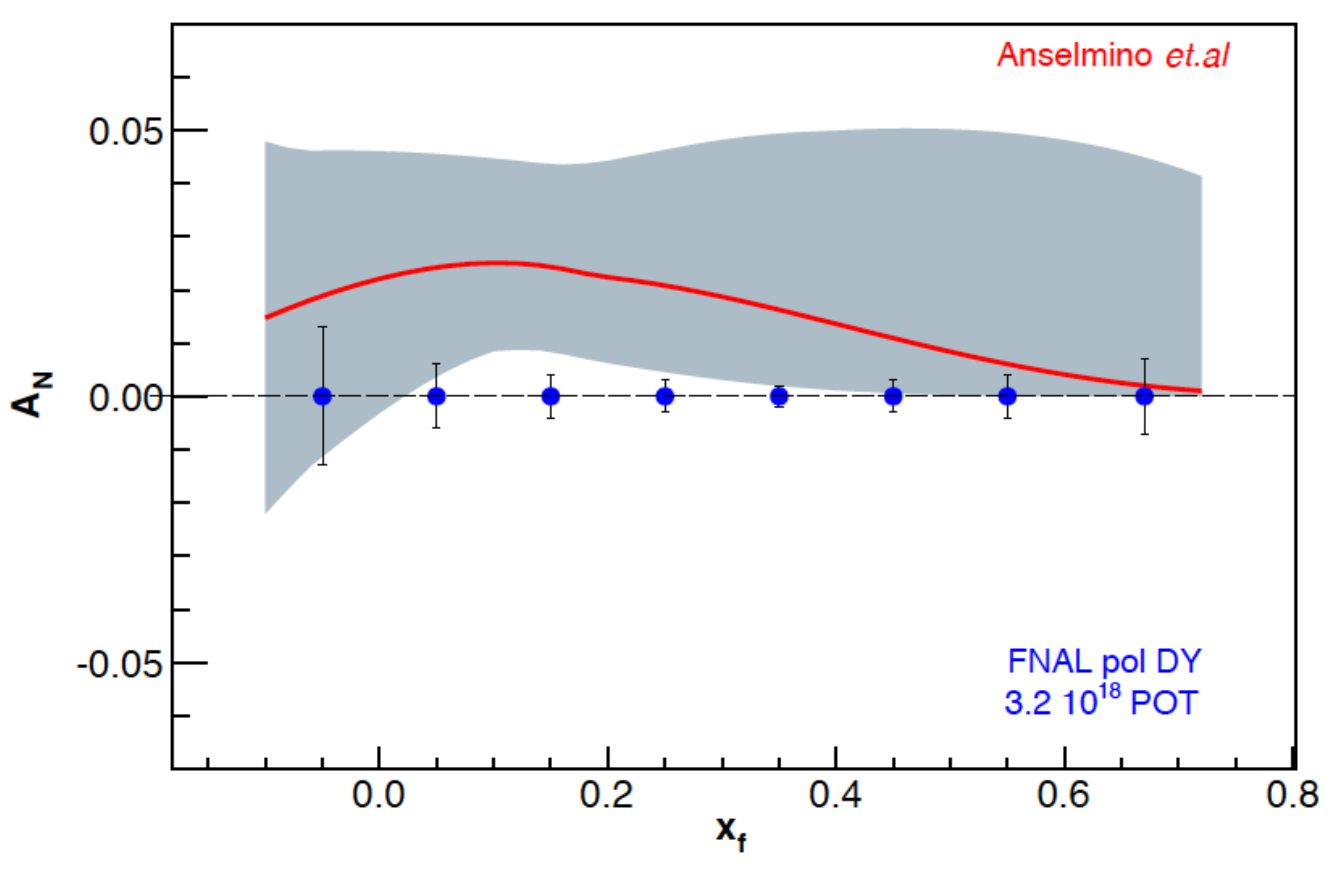




\section{TMDs probed via DY at Fermilab}

Boer-Mulders functions:

- Unpolarized Drell-Yan: $d \sigma_{D Y} \propto h_{1}^{\perp} \bar{h}_{1}^{\perp} \cos (2 \phi)$

E906, E1039, E1027

Sivers functions:

- Single transverse spin asymmetry in polarized Drell-Yan:

$A_{N}^{D Y} \propto f_{1 T}^{\perp}\left(x_{q}\right) f_{\bar{q}}\left(x_{\bar{q}}\right)$

E1039, E1027

Transversity distributions:

- Double transverse spin asymmetry in polarized Drell-Yan:

$A_{T T}^{D Y} \propto h_{1}\left(x_{q}\right) h_{1}\left(x_{\bar{q}}\right)$

E1027

- Drell-Yan and SIDIS involve different combinations of TMDs

- Drell-Yan does not require knowledge of the fragmentation functions

- T-odd TMDs are predicted to change sign from DIS to DY

(Boer-Mulders and Sivers functions)

Remains to be tested experimentally! $\rightarrow$ COMPASS, RHIC, EIC/SpinQuest for sea quarks 


\section{Summary and Outlook}

\begin{tabular}{|c|c|c|c|c|}
\hline Experiments & Run Time & $\begin{array}{l}\text { Collision } \\
\text { Types }\end{array}$ & Physics & 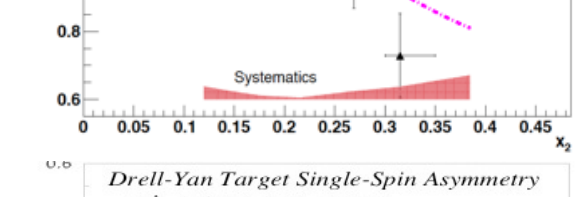 \\
\hline E906 & 2012-2017 & $\begin{array}{l}p+\text { targets } \\
(H, D, C, F e, \\
W)\end{array}$ & $\begin{array}{l}\text { - dbar/ubar asymmetry } \\
\text { - quark dE/dx }\end{array}$ & \\
\hline E1039 & $2018-2021+$ & $\begin{array}{l}\mathrm{p}+\text { pol. } \\
\text { targets } \\
\left(\mathrm{NH}_{3}, \mathrm{ND}_{3}\right)\end{array}$ & Sea-quark Sivers, TMDs & $\begin{array}{c}c^{0.7} \\
10^{-2} \\
10^{-4}\end{array}$ \\
\hline $\begin{array}{l}\text { E1067(para.) } \\
\text { DarkQuest }\end{array}$ & $\begin{array}{l}2017-2021+(\text { para. }) \\
2021+(\text { dedicated })\end{array}$ & $\begin{array}{l}p+\text { any } \\
\text { targets }\end{array}$ & $\begin{array}{l}\text { dark photon, dark } \\
\text { Higgs, ALP ... }\end{array}$ & $\begin{array}{c}10^{-6} \\
10^{-7} \\
10^{-8} \\
10^{-19} \\
1^{-2}\end{array}$ \\
\hline E1027 & $202 x$ & $\begin{array}{l}\text { Pol. p- } \\
\text { beam + }\end{array}$ & $\begin{array}{l}\text { - quark Sivers } \\
\text { - TMD, spin }\end{array}$ & \\
\hline $5 / 13 / 19$ & \multicolumn{3}{|c|}{ Ming Liu @QCD Evolution 2019} & \\
\hline
\end{tabular}




\section{SpinQuest/E1039 Collaboration}

\section{Collaboration}

INSTITUTION

Argonne National Laboratory great opportunities for new comers to contribute and lead major detector and physics efforts
Fermi National Accelerator Laboratory

KEK

Los Alamos National Laboratory

Richard Tesarek (PI), Carol Johnstone, Charles Brown

Shin'ya Sawada (PI)

Kun Liu (SP), Mikhail Yurov, Chun-Min Jen, Ming Liu, Xuan Li, Walter Sondheim

Lamiaa El Fassi (PI)

Stephen Pate (PI), Vassili Papavassiliou,

Haiwang Yu, Forhad Hossain

Yuji Goto (PI)

Kenichi Nakano (PI), Toshi-Aki Shibata

University of Colorado, Boulder

University of Illinois, UrbanaChampaign

University of Michigan

University of New Hampshire

University of Virginia

Jen-Chieh Peng (PI), Yen-Chu Chen

Wolfgang Lorenzon (PI), Minjung Kim, Noah Wuerfel

\section{FULL MEMBERS}

Donald Isenhower (PI), Michae Daugherity, Shon Watson

Paul Reimer (PI), Donald Geesaman

Edward Kinney (PI)

AFFILATE MEMBERS

Haley Stien, John Marsden, Mitchell

Schneller, Nathan Rowlands, Roy Salinas, Rusty Towell, Shannon McNease, Yves Ngenzi, Thomas Fitch

Kawtar Hafidi, Kevin Bailey, Thomas O'Connor, Zhihong Ye, Benjamin Zeidman

Robert Bushek, Dave Christian, Donald Mitchell, David Northacker, Mike Geelhoed, Kathy Graden, Bridget Iverson, van Vitev, Jin-Yuan Wu, Maddie Schoell, Steven Timm, Yanqiu Yin

Shigeru Ishimoto

an Boissevain, Melynda Brooks, Matt

Durham, David Kleinjan, Sho Uemura,

Cesar Da Silva, Patrick McGaughey, And Klein

Dipangkar Dutta Jumper, Jason Dove, Mingyan Tian, Bryan Dannowitz, Randall McClellan, Shivangi Prasad

Daniel Morton, Richard Raymond, Marshall Scott

Maurik Holtrop

Donal Day, Donald Crabb, Jixie Zhang, Oscar Rondon, Liliet Diaz, Arthur Conove Brandon Kriesten, Simonetta Liuti, Ellen Brown, Blaine Norum, Matthew Roberts

Takahiro Iwata, Norihiro Doshita 
backup 


\section{SpinQuest Experimental Hall}

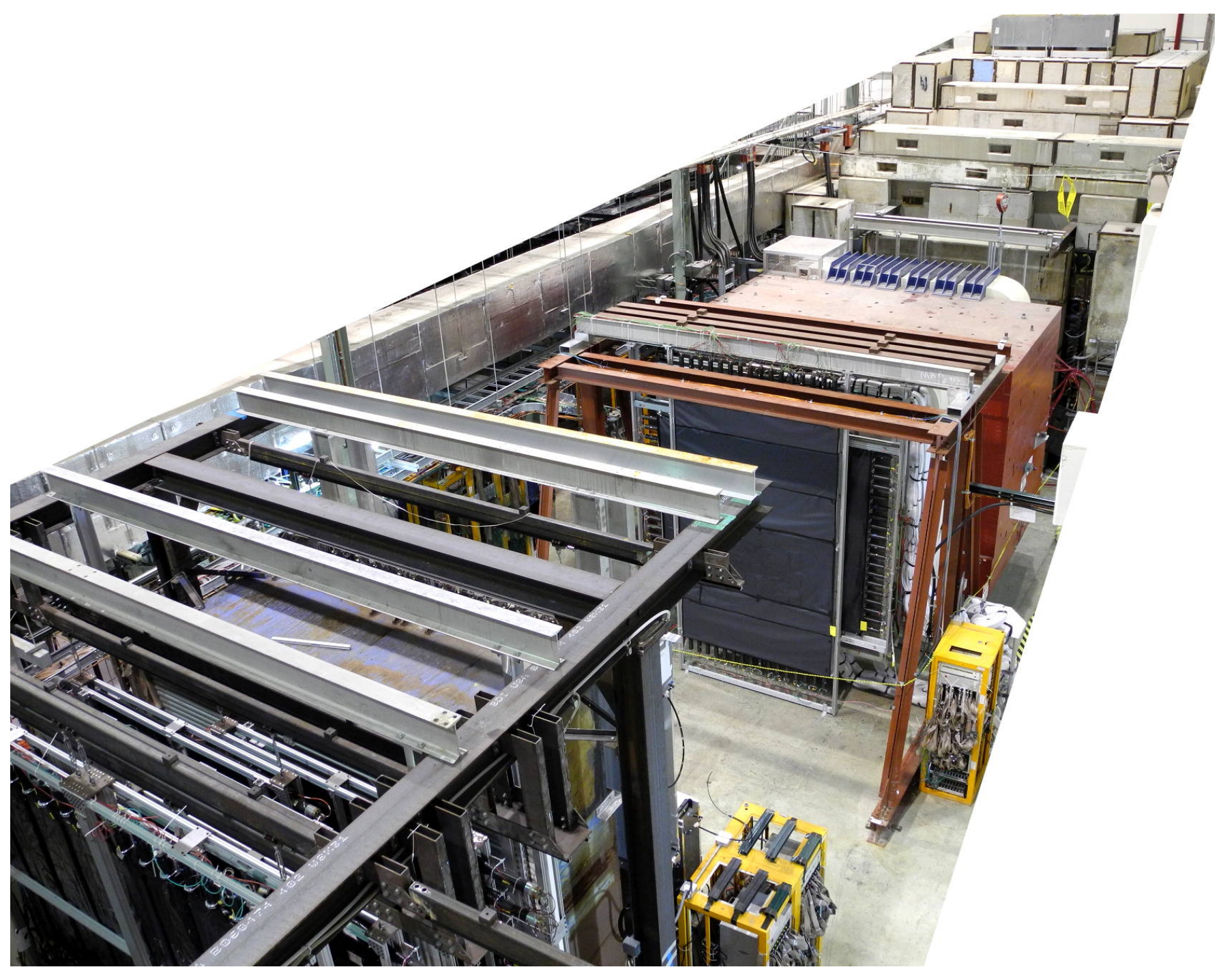

Target area

F-Mag

K-Mag 


\section{New Beam Collimator and Target}

Target cross section: $18 \times 28 \mathrm{~mm}^{2}$

Beam cross section:

Need be well contained within 4 sigma, required by $\mathrm{dR}<2 \times 10^{-4}$

$\operatorname{sig} X=18 / 2 / 4=2.2 \mathrm{~mm}$

$\operatorname{sigY}=28 / 2 / 4=3.5 \mathrm{~mm}$

Beam jitter: $d X=d Y \sim 1 \mathrm{~mm}$

$$
\begin{aligned}
& 1 \text { sig }=0.68269 \\
& 2 \text { sig }=0.95450 \\
& 3 \text { sig }=0.99730 \\
& 4 \text { sig }=0.99994
\end{aligned}
$$

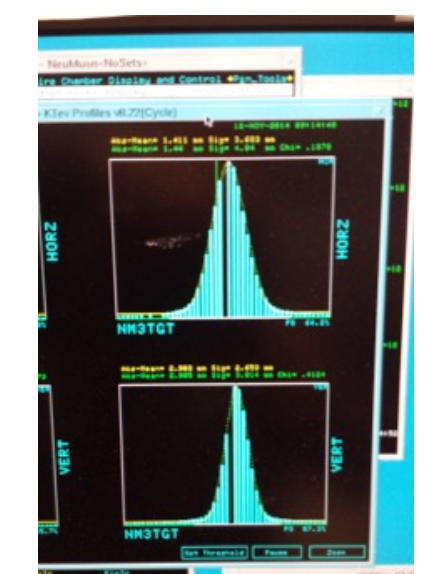

E906 beam profile:

SigX $=4.0 \mathrm{~mm}$

SigY $=3.0 \mathrm{~mm}$

$f(x, \mu, \sigma)=\frac{1}{\sigma \sqrt{2 \pi}} e^{-\frac{(x-\mu)^{2}}{2 \sigma^{2}}}$

Beam collimator
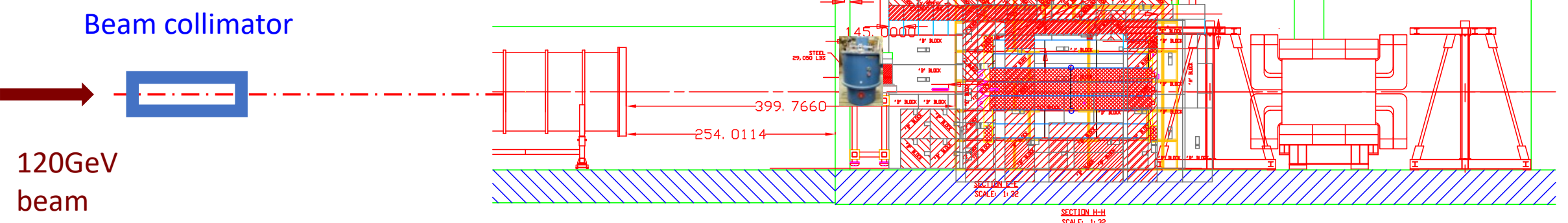


\section{Target and Beam Dump Event Separation target at upstream: $Z=-3.5 m$}

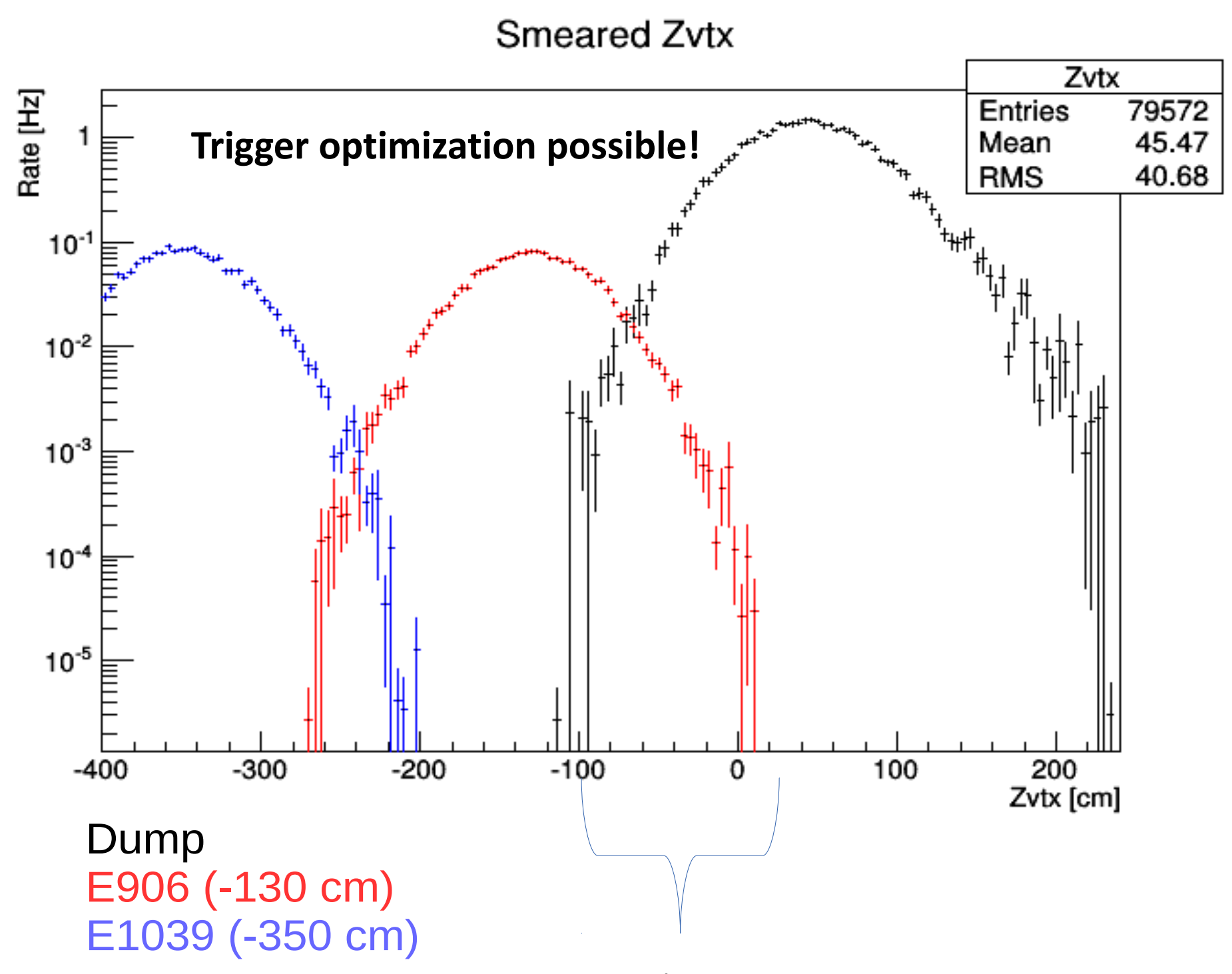




\section{Projected Dark Sector Physics Search Sensitivity}
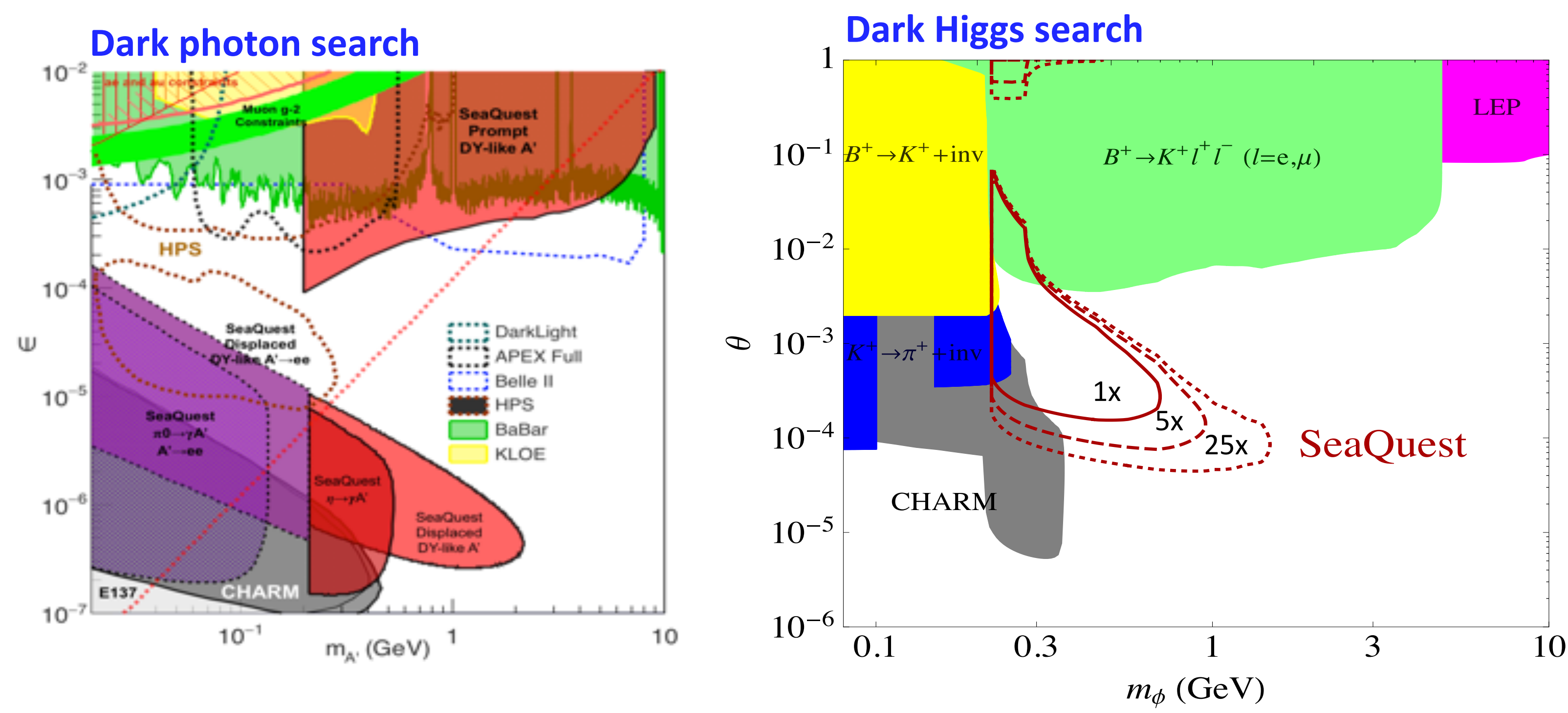